Ludger Heidbrink • Claus Langbehn Janina Loh Herausgeber

\title{
Handbuch Verantwortung
}

mit 13 Abbildungen und 1 Tabelle

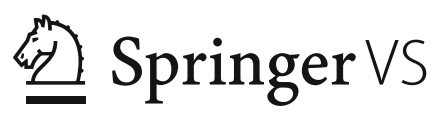


Herausgeber

Ludger Heidbrink

Christian-Albrechts-Universität zu Kiel

Kiel, Deutschland

Janina Loh

Universität Wien

Wien, Österreich

\author{
Claus Langbehn \\ Humboldt-Universität zu Berlin \\ Berlin, Deutschland
}

ISBN 978-3-658-06109-8

ISBN 978-3-658-06110-4 (eBook)

ISBN 978-3-658-06121-0 (Bundle)

DOI 10.1007/978-3-658-06110-4

Die Deutsche Nationalbibliothek verzeichnet diese Publikation in der Deutschen Nationalbibliografie; detaillierte bibliografische Daten sind im Internet über http://dnb.d-nb.de abrufbar.

\section{Springer VS}

(C) Springer Fachmedien Wiesbaden GmbH 2017

Das Werk einschließlich aller seiner Teile ist urheberrechtlich geschützt. Jede Verwertung, die nicht ausdrücklich vom Urheberrechtsgesetz zugelassen ist, bedarf der vorherigen Zustimmung des Verlags. Das gilt insbesondere für Vervielfältigungen, Bearbeitungen, Übersetzungen, Mikroverfilmungen und die Einspeicherung und Verarbeitung in elektronischen Systemen.

Die Wiedergabe von Gebrauchsnamen, Handelsnamen, Warenbezeichnungen usw. in diesem Werk berechtigt auch ohne besondere Kennzeichnung nicht zu der Annahme, dass solche Namen im Sinne der Warenzeichen- und Markenschutz-Gesetzgebung als frei zu betrachten wären und daher von jedermann benutzt werden dürften.

Der Verlag, die Autoren und die Herausgeber gehen davon aus, dass die Angaben und Informationen in diesem Werk zum Zeitpunkt der Veröffentlichung vollständig und korrekt sind. Weder der Verlag noch die Autoren oder die Herausgeber übernehmen, ausdrücklich oder implizit, Gewähr für den Inhalt des Werkes, etwaige Fehler oder Äußerungen. Der Verlag bleibt im Hinblick auf geografische Zuordnungen und Gebietsbezeichnungen in veröffentlichten Karten und Institutionsadressen neutral.

Gedruckt auf säurefreiem und chlorfrei gebleichtem Papier

Springer VS ist Teil von Springer Nature

Die eingetragene Gesellschaft ist Springer Fachmedien Wiesbaden GmbH

Die Anschrift der Gesellschaft ist: Abraham-Lincoln-Strasse 46, 65189 Wiesbaden, Germany 


\title{
Verantwortung, Rationalität und Urteil
}

\author{
Garrath Williams
}

\section{Inhalt}

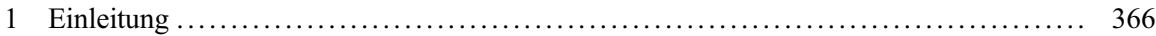

2 Hintergrund: Übergreifende Motive für die Verbindung von Verantwortung und

Rationalität (bzw. normativen Überlegungen im weiteren Sinne) .................. 367

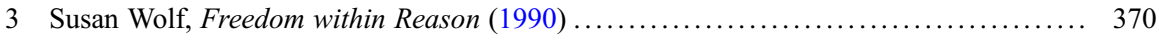

4 R. Jay Wallace, Responsibility and the Moral Sentiments (1994) .................. 373

5 Fischer und Ravizza, Responsibility and Control: A Theory of Moral Responsibility (1998) ...................................................... 377

6 Angela M. Smith: Verantwortung liegt im Urteil, nicht in der Wahl ............... 382

7 Pamela Hieronymi: Verantwortliche Tätigkeit als „Wertungskontrolle“............... 385

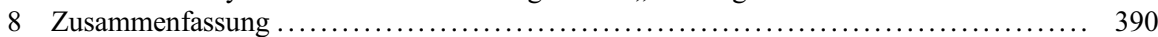

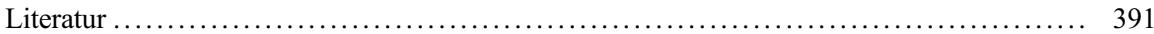

\section{Zusammenfassung}

Dieser Beitrag untersucht die Gründe, die dafür sprechen, Verantwortung mit den Fähigkeiten des moralischen Denkens und Urteilens in Verbindung zu bringen. Er stellt fünf verschiedene Ansätze vor, die Verantwortung und Rationalität miteinander verknüpfen: die Arbeiten von Susan Wolf, R. Jay Wallace, Angela M. Smith und Pamela Hieronymi sowie die Gemeinschaftsarbeit von John Martin Fischer und Mark Ravizza. Am Leitfaden dieser Autoren wird gezeigt, dass der Begriff des rationalen Vermögens zentral ist für das Verständnis und die Begründung unserer Verantwortungspraxis. Die Debatte über diesen Zusammenhang hat bereits deutliche Fortschritte gezeitigt, doch wirft der Begriff eines rationalen oder moralischen Vermögens nach wie vor erhebliche Verständnisprobleme auf. Ein denkbarer Grund hierfür, der erörtert wird, ist der, dass dieses Vermögen in

\footnotetext{
Übersetzung: Katrin Grünepütt

G. Williams ( $\square)$

Department of Politics, Philosophy and Religion, Lancaster University, Lancaster, Großbritannien

E-Mail: g.d.williams@lancaster.ac.uk
} 
einem konstitutiven Verhältnis zur Praxis des Zur-Verantwortung-Ziehens und Verantwortung-Übernehmens stehen könnte - und dass dieses Verhältnis noch gründlicher Erforschung harrt.

\section{Schlüsselwörter}

Fähigkeit • Gründe • (Handlungs-, Selbst-)Kontrolle • Moral • Rationalität • Rechenschaft $\bullet$ Urteil

\section{$1 \quad$ Einleitung}

Viele Arbeiten zur Verantwortung weisen dem menschlichen Vermögen der Rationalität einen besonderen Rang zu. Dies geschieht meist im Hinblick auf solche Fähigkeiten, die ein Individuum für seine Handlungen verantwortlich machen. Eine darüber hinausgehende, hier nicht im Mittelpunkt stehende Frage wäre die nach der Rationalität der Praxis selbst, aufgrund deren wir einander als verantwortliche Subjekte behandeln.

Das Urteilsvermögen hat demgegenüber eine weniger wichtige Rolle in der Debatte gespielt, ist aber ebenfalls bedeutsam. Es kommt dann zum Zuge, wenn ein verantwortungsfähiger Mensch seine Situation erfasst, Handlungsmöglichkeiten abwägt und schließlich tatsächlich handelt. Nach manchen Autoren manifestieren Urteile sich auch in unseren spontanen Reaktionen und unseren Aufmerksamkeitsmustern (einschließlich dessen, was unserer Aufmerksamkeit entgeht). Außerdem wirkt das Urteilsvermögen natürlich mit beim Rückblick auf Handlungen und ihre Folgen, bei der Zuschreibung und Zumessung von Verantwortung für sie und bei der Entscheidung über das Ob bzw. Wie einer Reaktion auf sie.

Für die Zwecke dieses Beitrags ist es nützlich, Rationalität und Urteil begrifflich zusammenzuspannen, da nicht jeder Philosoph von sich aus normative Überlegungen im Sinne von Rationalität verstehen wird. ,Normativ "verwende ich hier als Sammelbegriff für die Forderungen der Klugheit, der Moral und auch der sozialen Rollen. Dass Klugheitserwägungen eine Sache der Rationalität sind, steht außer Zweifel, auch wenn ungewiss bleibt, ob es in der Frage der Gewichtung von kurzgegenüber langfristigen Aspekten eine ,rationale' Entscheidung geben kann. Hingegen wird von etlichen Philosophen bezweifelt, dass Moral und erst recht soziale und institutionelle Verhaltenserwartungen als auf Vernunft bzw. unserem rationalen Vermögen gegründet gedacht werden können. Über zweierlei dürfte indes Einigkeit bestehen: Erstens ist es für verantwortungsfähige Wesen sowohl möglich als auch bisweilen notwendig, über normative Ansprüche nachzudenken, gleich, ob diese Ansprüche Fragen der Klugheit, der Moral oder der sozialen Erwartung berühren. Und zweitens kann keine Moraltheorie, wie wenig Gewicht sie auch immer der Rationalität beimisst, die Schlüsselrolle des Urteilens im praktischen Leben verleugnen. Kurz, mit Hilfe des Begriffspaars ,Rationalität und Urteil' lässt sich auf die Rolle moralischer und anderer normativer Überlegungen im Agieren und Interagieren verantwortlich Handelnder hinweisen. 
Der nachstehende Beitrag ist folgendermaßen gegliedert: Einleitend werden die naheliegenden Gründe für die Verbindung von Rationalität und Verantwortung genannt, und es wird an Harry Frankfurts bekannte Konzeption der Handlungsfreiheit erinnert - eine Konzeption, die justament deshalb scheitert, weil sie Rationalität und moralisches Urteil ausklammert. Im Hauptteil werden dann in mehr oder weniger chronologischer Reihenfolge fünf Ansätze vorgestellt, die das rationale Vermögen verantwortlich Handelnder thematisieren. Jeweils einen eigenen Abschnitt erhalten dabei Susan Wolfs Freedom within Reason (Wolf 1990), R. Jay Wallaces Responsibility and the Moral Sentiments (Wallace 1994) und die Gemeinschaftsarbeit von John Martin Fischer und Mark Ravizza, Responsibility and Control (Fischer und Ravizza 1998). Die letzten beiden Abschnitte gelten zwei Autorinnen, die in den vergangenen zwölf Jahren mehrere bedeutende Arbeiten über verantwortliches Handeln vorgelegt haben und deren Auffassungen beträchtliche Parallelen aufweisen: Angela M. Smith und Pamela Hieronymi. Wie sich zeigen wird, gibt es für die Verknüpfung der Verantwortung mit dem Denk- und Urteilsvermögen zwingende Gründe, doch wirft der dafür notwendige Begriff des Vermögens bzw. der Fähigkeit (,ability“) grundsätzliche Fragen bezüglich der Natur des moralischen Handelns und der Moralgemeinschaft auf.

\section{$2 \quad$ Hintergrund: Übergreifende Motive für die Verbindung von Verantwortung und Rationalität (bzw. normativen Überlegungen im weiteren Sinne)}

Zwischen Rationalität und Verantwortung besteht offensichtlich ein grundlegender Zusammenhang. Die Praxis des Zur-Verantwortung-Ziehens zeichnet Verhältnisse zwischen erwachsenen Menschen aus. Sie verdankt sich der dem Menschen eigentümlichen Fähigkeit, Rechenschaft über sein Handeln abzulegen, dasselbe auch von seinesgleichen zu verlangen und einige dieser Handlungen und Rechtfertigungen als unvertretbar abzulehnen. Der Zusammenhang schlägt sich nicht zuletzt sprachlich nieder: In ,Verantwortung ' steckt das Antworten, das Rede-und-Antwort-Stehen, so wie engl. ,responsibility` das Verb ,to respond' enthält und im sinnverwandten

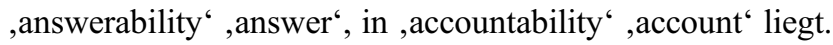

Damit soll keineswegs bestritten werden, dass diese menschliche Fähigkeit gewisse Vorformen in den Beziehungen zwischen anderen intelligenten Tieren besitzt, die sich ein ihnen nützliches Verhalten gern gefallen lassen und sogar erwidern, ihnen zugefügten Schaden hingegen mit Unwillen aufnehmen und sogar vergelten. Da diese Tiere aber weder ihr Tun und Lassen nebst seinen Beweggründen in Worte zu fassen noch Einwände gegen das Verhalten anderer oder gar normative Ansprüche zu artikulieren vermögen, können wir in ihrem Falle nur von Reaktionen und nicht von Antworten/Verantwortung sprechen, so wenig wie von Zurechnen/ Zurechenbarkeit oder von Rechenschaft.

Der grundlegende Zusammenhang von Rationalität und Verantwortung weist auch auf die zentrale Bedeutung normativer Maßstäbe für die Frage der Verantwortung 
hin. Es trifft zwar zu, dass wir jemandem Verantwortung für eine Tat oder deren Folgen zuschreiben können, ohne dabei schon ein moralisches oder sonstwie normatives Urteil zu fällen. Unsere Aussage beschränkt sich in diesem Falle darauf, dass der Betreffende etwas bestimmtes getan oder eine bestimmte Folge herbeigeführt habe, dass also Tat oder Folge Niederschlag seines Handlungsvermögens - und nicht etwa von zwingender Notwendigkeit, einer gestörten Geistesverfassung oder einem merkwürdigen Zufall - sei. (Auch dann ist freilich immer noch eine Art normativen Urteils im Spiel, insofern wir urteilen oder jedenfalls davon ausgehen, dass der Betreffende über die für verantwortliches Handeln notwendigen Fähigkeiten verfüge. Jedoch müssen wir in einem solchen Fall weder den Handelnden noch seine Tat oder deren Folge als gut oder schlecht bewerten.) Meist beziehen wir uns aber, wenn wir jemanden als verantwortlich für eine Tat oder deren Folge bezeichnen, auf die handlungsleitenden Normen: Jemanden für verantwortlich zu halten, impliziert üblicherweise, sowohl ihn als Handelnden wie auch sein Handeln zu bewerten - negativ bei Tadel und Bestrafung, positiv bei Lob und Lohn. Die Frage, ob unsere Bewertungsmaßstäbe ihrerseits letztlich der Vernunft entspringen, kann, wie bereits angedeutet, im vorliegenden Rahmen vernachlässigt werden. Entscheidend ist, dass die Verantwortungspraxis in der Regel die Existenz moralischer und anderer Normen voraussetzt; dass über diese Normen nachgedacht werden kann und de facto nachgedacht wird; dass es stets eine Urteilsfrage ist, inwieweit die Normen in einer gegebenen Situation gelten; und dass wir auch über diese Urteilsfrage nachdenken können.

Nicht selten wird der fundamentale Zusammenhang von Rationalität und Verantwortung durch Debatten über Determinismus und Verantwortung verdunkelt, in denen recht unpräzis von der Freiheit bzw. „Fähigkeit, anders zu handeln“, die Rede zu sein pflegt. Diese Fähigkeit, das sollte allseits einsichtig sein, bedarf der Spezifikation. Einfach zu sagen, jemandes Handeln, Denken oder Urteilen sei ,nicht determiniert ${ }^{`}$, reicht, wie bereits Hume und Kant gewusst haben, nicht aus, um zu verstehen, was verantwortliches Handeln sei: Vielleicht ist ja das Universum indeterministisch, oder es gibt spezielle naturgesetzliche Strukturen, aufgrund deren Handlungsfolgen nicht determiniert sind. Verantwortliches Handeln, was immer es auch sein mag, ist jedenfalls kein schlichtes Zufallsprodukt. Sein Träger muss als in irgendeiner Weise selbstbestimmt verstanden werden. Anders gesagt: Das Problem, wie wir Personen als Urheber ihrer Handlungen sowie der diesen Handlungen zugrunde liegenden Gedanken und Urteile verstehen sollen, besteht unabhängig davon, wie wir Freiheit und Verantwortung genau zueinander ins Verhältnis setzen.

Zum Hintergrund der vorzustellenden neueren Debatten gehört auch die Position Harry Frankfurts, die darum hier kurz in Erinnerung gerufen sei. Laut Frankfurt (Frankfurt 1971) beruht der menschliche Wille auf unserer Fähigkeit, uns mit bestimmten Wünschen zu identifizieren, indem wir „Wünsche zweiter Stufe“ (,second-order desires“) ausbilden derart, dass wir bestimmte Dinge „zu wünschen wünschen“ (,want to want") bzw. „,nicht zu wünschen wünschen“" (,want not to want"). Der Wille ist dann im Wesentlichen die Fähigkeit, in Bezug auf einen Wunsch $z u$ 
handeln zu wünschen. Die Mutter, die einverstanden ist mit ihrer Mutterschaft, ,wünscht" in diesem Sinne die Mehrzahl der damit verbundenen Pflichten ,zu erfüllen zu wünschen', wohingegen die Drogensüchtige wider Willen es ,wünscht", ihrer Sucht ,nicht nachzugeben zu wünschen'. Zum Thema ,Verantwortung' sagt Frankfurt nur sehr wenig, aber sein Grundgedanke ist der, dass Willensfreiheit aus dem Einklang zwischen erst- und zweitstufigen Wünschen entsteht und nicht aus kausaler Nicht-Determination.

Frankfurts Konzeption mag als nicht-metaphysische Freiheitstheorie ihren Reiz haben, sie sieht sich jedoch entscheidenden Einwänden gegenüber. Einer davon besagt, dass sie uns kein Kriterium für Verantwortung an die Hand gibt. Es ist beispielsweise denkbar, dass ich ,wünsche, nicht zu wünschen', mich an meiner ehemaligen Geliebten zu rächen. Dieser „Wunsch zweiter Stufe“ enthebt mich aber nicht der Verantwortung, wenn ich dann trotzdem rachsüchtig handele. Tatsächlich könnten meine inkompatiblen Wünsche mich sogar eher zur Selbsttäuschung als zur Selbstbeherrschung führen, etwa indem ich meine eifersüchtige Einmischung rationalisierend für einen Ausdruck legitimer Sorge halte. (Dieses Problem wird bei Frankfurt dadurch verdeckt, dass er sich auf Beispiele suchtgesteuerter oder zwanghafter Wünsche konzentriert, die Verantwortung grundsätzlich zu unterminieren scheinen.) Noch schwerer ins Gewicht fällt in unserem Kontext ein zweiter Einwand, den Gary Watson in seinem Aufsatz ,Free Agency ${ }^{6}$ (Watson 1975) vorgetragen hat. Ihm zufolge geht Frankfurts Theorie insgesamt nicht auf, weil sie dem Denken und den Werten keinen angemessenen Platz zubilligt. Denn die Überlegung, die ein verantwortungsfähiger Mensch anstelle, sei keine das Faktische betreffende (wünscht er oder wünscht er nicht, einen bestimmten Wunsch zu haben?), sondern eine normative (soll ein bestimmter Wunsch verwirklicht oder soll er verworfen werden?). „Man fragt sich für gewöhnlich nicht - oder braucht sich nicht zu fragen -, welchen seiner Wünsche man handlungswirksam sehen möchte; man fragt sich, welche Handlungsweise die beste sei. Die praktische Ausgangsfrage betrifft Handlungsweisen und nicht einen selbst [sprich: die Wünsche, die man hat]", schreibt Watson (Watson 1975, S. 219). Frankfurts Ansatz unterschlägt mit anderen Worten die Fähigkeit verantwortlicher Menschen zum rationalen Handeln. Er scheitert sowohl als Freiheitswie als Verantwortungskonzeption.

Ein Anliegen der im Folgenden betrachteten Autoren ist es, der Fähigkeit zum rationalen Handeln einen Stellenwert einzuräumen, ohne es in einer Weise zu interpretieren, die mit einem deterministischen Weltbild wesensmäßig unvereinbar wäre. Dies nicht in erster Linie deshalb, weil unsere Autoren Anhänger des Determinismus wären, sondern vielmehr aus dem bereits erwähnten Grunde, dass bloße Nicht-Determiniertheit uns im Verständnis dessen, was die Fähigkeit zum verantwortlichen Handeln auszeichnet, nicht weiterhilft. Beiträge zur Problemlösung liefern alle Positionen, wobei ein beständiger Fortschritt zu verzeichnen ist und die Arbeiten von Angela Smith und Pamela Hieronymi uns m. E. einem zufriedenstellenden Verständnis der Problematik am nächsten bringen. 


\section{Susan Wolf, Freedom within Reason (1990)}

Susan Wolf stellt ihre Konzeption als Antwort auf zwei einander ausschließende Auffassungen von moralischer Verantwortung vor. Bei der einen von ihnen, von Wolf ,Wahres Selbst'-Theorie genannt, handelt es sich erkennbar um diejenige Harry Frankfurts, die Wolf allerdings unter dem Eindruck von Watsons Einwand, wonach die Werte eines Handelnden ebenso maßgebend sind wie seine Wünsche, zu plausibilisieren sucht. Ein Mensch ist demzufolge ,frei und verantwortlich für sein Verhalten dann und nur dann, wenn sein Verhalten durch seinen Willen und sein Wille durch seine Wünsche gesteuert werden kann [...]. Die für Verantwortung erforderliche Freiheit [...] ist [...] die Freiheit, das $\mathrm{zu}$ tun, was das innerste, eigentliche oder wahre Selbst wünscht - was freilich etwas anderes sein kann als das, wozu die stärksten Wünsche einen drängen [...] [Somit] hängt des Menschen Status als freies und verantwortliches Wesen nicht daran, $o b$, sondern daran, wie seine Handlungen determiniert sind“" (aus Wolfs späterer Zusammenfassung ihrer Monografie - 2005, S. 265 [Kursiven sind die der Übers.]). Dagegen geht die andere, die ,Autonomie'-Theorie, davon aus, dass Lebewesen ,,verantwortlich nur insoweit [sind], als sie autonom sind. Werden ihre Handlungen von außerhalb ihres Selbst liegenden Kräften gesteuert oder wird gar ihr Selbst von ihm äußerlichen Kräften gesteuert, so sind sie für die daraus folgenden Handlungen nicht verantwortlich“ (Wolf 2005, S. 261). Es liegt auf der Hand, dass eine solche Sicht der Dinge häufig eine inkompatibilistische Verantwortungskonzeption begründet, wo ihr nicht schon gleichkommt.

Wolfs Einwand gegen die ,Autonomie'-Theorie ist ein zum Teil altvertrauter. Die Theorie lehnt die Annahme, jemandes Handlungen und sonstige Eigenschaften seien determiniert, mit der Begründung ab, sie lasse keinen Spielraum für die individuelle Verantwortung. Wenn aber Autonomie gleichbedeutend damit sein soll, dass jemandes Gedanken und Handlungen bloß nicht-determiniert sind, ist schwer erkennbar, in welchem Sinne dann noch gesagt werden kann, dass eine Person hinter den Handlungen stehe (Wolf 1990, S. 14). Wolf spitzt diesen bekannten Punkt noch zu, indem sie darauf hinweist, dass in der logischen Konsequenz dieser Theorie Autonomie als Fähigkeit verstanden werden müsste, eine Wahl zu treffen ohne jegliche Grundlage, d. h. unabhängig von den Empfehlungen der Vernunft oder des Begehrens (Wolf 1990, S. 53 f.). Was aber, so fragt Wolf, sollte der Sinn oder Wert eines solchen nihilistischen Vermögens sein?

Wolfs Argumentation gegen die ,Wahres Selbst'-Theorie ist von komplexer Art und stützt sich stark auf Überlegungen, die die Autorin bereits in einer früheren Arbeit angestellt hat (Wolf 1987, vgl. a. Wolf 1990, S. 37 f., 44, 75 ff., Wolf 2005, S. 266 ff.). Demnach ist ein Mensch, der aufgrund seiner Sozialisation abstoßende Werte hegt und außerstande ist, Gründe für andere Denk- und Handlungsweisen anzuerkennen, recht besehen für sein eigenes Denken und Verhalten nicht verantwortlich, obwohl es offenkundig sein ,wahres' Selbst repräsentiert. Einen solchen Menschen sollten wir nicht tadeln, denn er ist letzten Endes nicht ,zurechnungsfähig“ - es fehlt ihm „,die Fähigkeit, Recht von Unrecht zu unterscheiden“ (Wolf 1987, S. 56). Als zweiten Haupteinwand gegen, Wahres Selbst‘-Theorien führt Wolf 
an, dass sie Urteile über Verantwortlichkeit nicht in ihrer ganzen „Tiefe“ zu erfassen vermöchten. Zu meinen, Menschen drückten in ihrem Handeln - jedenfalls wenn sie freiwillig und nicht unter Zwang handeln - schlichthin ihr, wahres Selbst' aus, führe dazu, dass man in ihnen nurmehr ,schlechte“, ,gute“ oder irgendwo zwischen diesen beiden Polen einzuordnende „Tat-Urheber“ sehe (Wolf 1990, S. 39). Aussagen über Verantwortung erschöpften sich aber nicht darin, Menschen in dieser Weise einzustufen (um einen von Smart 1961 geprägten Begriff zu entlehnen): Sie seien „spezifischer, nicht-instrumentell und offenbar auch gewichtiger“" (Wolf 1990, S. 41). Diesem Einwand ließe sich im Rahmen der,Wahres Selbst'-Theorien nach Wolf nur begegnen, wenn man zu erklären wüsste, wie Personen für ihr Selbst verantwortlich sein könnten. Indes schließt unser Bewusstsein vom Einfluss der Erziehung und Gesellschaft eine solche Möglichkeit von vornherein aus, ganz zu schweigen von den Problemen, die sich mit der ,Autonomie'-Theorie verbinden. (Dieser Aspekt soll im Folgenden weitgehend außer Betracht bleiben. Er steht nicht im thematischen Zentrum dieses Beitrags, kommt allerdings als Bedenken gegen ,attributionistische“ Darstellungen wie die von Angela Smith zum Tragen: siehe weiter unten Abschn. 6.)

Weil beide Theorien eine Eigenschaft voraussetzen, „die sich auf eine nicht, auch nicht implizit, wertende Art beschreiben lässt [sc. Freiheit von Kausaldetermination; Fähigkeit, seinen Wünschen oder Werten entsprechend zu handeln], verfehlen sie das Hauptmerkmal, durch das verantwortliche Wesen sich von anderen unterscheiden“", meint Wolf(Wolf 1990, S. 77). Wir sollten deshalb besser auf das menschliche Vernunftvermögen setzen, das heißt auf die Fähigkeit, gemäß einer rational vertretbaren Wertorientierung zu denken und zu handeln. Mit Blick auf die ,Autonomie'Theorien wäre es für die Feststellung verantwortlichen Handelns demnach nicht ausschlaggebend, dass das Handeln durch die Einflüsse, die einen Menschen geprägt haben, nicht kausal determiniert wird, sondern dass es determiniert wird durch die Fähigkeit des Betreffenden, im Einklang mit der Vernunft zu denken und zu handeln. Zwischen diesen beiden Arten der Determination besteht im Übrigen keinerlei Konkurrenz, erheischen sie doch völlig unterschiedliche Erklärungsansätze (Wolf 1990, S. 72). In Bezug auf die ,Wahres Selbst'-Theorien käme es wiederum darauf an, verantwortliches Handeln nicht als simple Resultante der Charakterzüge oder Fähigkeiten des jeweiligen Menschen zu verstehen, sondern einzusehen, dass, solange diese Fähigkeiten und Eigenarten nicht mit der Fähigkeit einhergehen, moralische Gesichtspunkte zu würdigen und ihnen gemäß zu handeln, verantwortliches Handeln gar nicht vorliegt. Nur wer Recht von Unrecht halbwegs zu unterscheiden vermag, ist ein Kandidat für moralische Verantwortung.

Freilich schafft diese ihre Konzeption, wie Wolf selbst herausstellt (Wolf 1990, S. 79 ff., vgl. a. 1980), eine Asymmetrie bei der Verantwortungszuschreibung. So wären Menschen, die sich fest bestimmten Formen des Unrechts verschrieben haben, nicht verantwortlich, da sie unfähig sind, Gründe für rechtschaffenes Denken und Handeln anzuerkennen. Andere hingegen, die sich fest dem ,Wahren und Guten" verschrieben haben und zum Beispiel einfach nicht lügen oder stehlen können, wären trotzdem ohne Abstriche zu loben. Denn der Prüfstein für die Zuschreibung von Verantwortung liegt nicht in der „Fähigkeit, anders zu handeln“, sondern in der „Fähigkeit, recht zu handeln“. Diese Asymmetrie ist nach beiden 
Seiten hin anfechtbar. Auf den Übeltäter, der unfähig oder jedenfalls in geringerem $\mathrm{Maße}$ fähig ist, recht zu handeln, kommen wir gleich zurück. Was soll man aber zu demjenigen sagen, der konstant recht handelt und behauptet, er könne gar nicht anders? Angenommen, dem wäre so - würde er dann kein Lob verdienen? Ich will hier nicht versuchen, diese Frage abschließend zu klären, sondern lediglich (mit Wolf) anmerken, wie befremdlich eine Annahme wäre, der zufolge die Fähigkeit, recht zu handeln, dann moralisch weniger profiliert sein würde, wenn sie absolut verlässlich zur Verfügung stünde. Es fällt schwer zu denken, dass bei den wenigen Menschen, auf die wir glauben unter allen Umständen bauen zu können, etwas anderes als Lob und Dankbarkeit am Platze sei (vgl. Williams 1993).

Mit der Fähigkeit, Recht von Unrecht zu unterscheiden und über den Unterschied zu reflektieren, hat Wolf unbestreitbar einen wesentlichen Punkt herausgearbeitet. Weniger eindeutig ist es aber, ob die Zuschreibung einer partiellen Unfähigkeit zu solchem Unterscheiden sich in der von Wolf gedachten Weise als Grundlage für die Praxis des Zur-Verantwortung-Ziehens eignet. Einige Menschen sind zweifellos so radikal unempfänglich für rationale Überlegungen, dass es sinnlos scheint, sie wie Verantwortliche $\mathrm{zu}$ behandeln - so, wie ja auch nicht-vernunftbegabte Entitäten nicht als verantwortlich behandelt werden. Dennoch gibt es Gründe, Bedenken gegen Wolfs leitende Intuition zu hegen, wonach jene, die weniger fähig sind, recht zu handeln, deshalb auch weniger verantwortlich für ihr Handeln sind.

Zunächst einmal ist die Zuschreibung eines moralischen oder rationalen Vermögens, wie auch Wolf einräumt (Wolf 1990, S. 87), problematisch und mit Unsicherheiten behaftet. Zwar gibt es natürlich einen Sinn, in dem sich sagen lässt, der ordentlich sozialisierte Rassist sei ,nicht fähig', von sich aus einen antirassistischen Standpunkt zu vertreten. Es ist aber anzunehmen, dass sich in den meisten Fällen doch Wege finden lassen werden, ihn für solche Gedanken zugänglich zu machen sei es mit Argumenten wie Fairness, Mitleid oder was auch immer. Da die Argumente für den Egalitarismus so elementar sind, ist es nie leicht zu beurteilen, inwieweit Fähigkeit oder echte Unfähigkeit, sie einzusehen, vorliegt.

Doch auch wenn wir einmal unterstellen, es ließen sich zumindest relative Aussagen über moralische Fähigkeit bzw. Unfähigkeit treffen, konfrontiert uns Wolfs vermeintlich intuitive Annahme mit kontraintuitiven Implikationen. Moralisch ,weniger fähige' Menschen handeln schlechter - und öfters schlechter - als andere. Gerade diejenigen, die wir also am stärksten zur Verantwortung ziehen müssten, sollen wir nach Wolfs Konzeption am wenigsten für verantwortlich halten. Um obiges Beispiel aufzunehmen, leuchtet es aber kaum ein, dass einer Unzugänglichkeit für antirassistische Argumente, einer Unempfänglichkeit für den Egalitarismus, einer Blindheit gegen das Menschsein bestimmter anderer Menschen exkulpatorische Kraft zukommen sollte. Ebenso gut kann man darin eine besonders tief sitzende - und umso tadelnswertere - moralische Verderbtheit sehen.

Ein verwandtes Problem tritt auf, wenn wir bedenken, dass viele Menschen sich exakt so lange anständig verhalten, wie sie im Einflussbereich anständiger gesellschaftlicher Institutionen leben, die ,soziales` oder, wie es manchmal auch genannt wird, ,prosoziales“ Verhalten belohnen und sein Gegenteil bestrafen. Kollabieren solche Institutionen oder werden von einer tyrannischen Macht usurpiert, können 
dieselben Menschen zu bereitwilligen Übeltätern werden und beispielsweise dazu übergehen, ihre Nachbarn zu verraten oder zu töten. Die Geschichte lehrt uns, dass dergleichen nicht so selten vorkommt. Vielleicht sollten wir daran festhalten, dass diese Menschen dem Übel ,hätten` widerstehen ,können'. (Wenn man für rechenschaftspflichtig nur den hält, der fähig ist, besser zu handeln, drängt sich eine entsprechende Fähigkeitszuschreibung geradezu auf.) Vielleicht könnten wir aber auch in Betracht ziehen, dass die betreffende Fähigkeit nicht so sehr eine Eigenschaft des Individuums simpliciter als vielmehr eine Eigenschaft des Individuums innerhalb einer bestimmten Bandbreite gesellschaftlicher Rahmenbedingungen ist. Diese Überlegung wird sehr schön bei Vargas ausgeführt (Vargas 2013, insbes. Kap. 6).

Es geht mir hier nicht darum, eine Entscheidung zwischen diesen beiden Möglichkeiten herbeizuführen. Der springende Punkt ihrer Gegenüberstellung ist erneut einfach der, dass der Begriff der Fähigkeit kein scharf umrissener ist und dass er Probleme der empirischen Zuschreibung aufwirft. Eine wichtige Implikation der zweiten Sichtweise möchte ich jedoch aufzeigen. Sie weist nämlich darauf hin, dass bei vielen (?) Menschen die Fähigkeit, moralischen Erwägungen zu folgen, sich zumindest teilweise der Tatsache verdankt, dass andere Menschen bereit sind, sie zur Verantwortung zu ziehen: mit Kritik und Widerstand zu reagieren, wenn sie etwa versuchen sollten, ihren Nachbarn Schaden zuzufügen; wertschätzend mit ihnen zu interagieren, sofern sie angemessene Rücksicht auf andere nehmen. Wenn aber das moralische Vermögen teilweise geformt und aufrechterhalten wird durch die Praxis des Zur-Verantwortung-Ziehens, kann es logischerweise nicht als unabhängige Basis für die Begründung dieser Praxis herhalten. Ja, wir könnten die These aufstellen, dass Behauptungen, wonach geistig zurechnungsfähige Erwachsene generell fähig sind, die Mitwirkung an Übeltaten - außer vielleicht in Extremsituationen des Terrors - zu verweigern, keine reinen Tatsachenbehauptungen sind, sondern immer auch eine konstitutive Dimension enthalten: das heißt, dass sie dazu beitragen, ein Bewusstsein unser selbst und dessen, was wir ,vermögen', zu wecken und insbesondere auch, die Bereitschaft anderer, uns verantwortlich zu machen, falls wir dem Bösen nicht widerstehen, zu antizipieren. Wenn Aussagen über moralische Fähigkeiten diese tatsächlich zugleich mitkonstituieren, erhält jener alte, vertrackte Grundsatz: „Sollen setzt Können voraus“ eine unerwartete neue Bedeutung.

\section{R. Jay Wallace, Responsibility and the Moral Sentiments (1994)}

Ein weiterer Ansatz, der das rationale Vermögen betont, ist der von R. Jay Wallace. Sein Buch verbindet zwei Gedankenstränge miteinander. Zum einen bezieht es sich auf Peter Strawsons berühmten Aufsatz ,Freedom and Resentment' (Strawson 1962; dt. ,Freiheit und Übelnehmen'), der die Rolle reaktiver Gefühle wie Übelnehmen, Empörung und Dankbarkeit in menschlichen Alltagsbeziehungen untersucht. Nach Strawson fungiert unsere Praxis des Zur-Verantwortung-Ziehens nicht als Instrument, mit dem wir einander manipulieren in der Hoffnung, ein wechselseitig gebilligtes Verhalten zu fördern (wie im utilitaristischen Verständnis von Verantwortung, 
z. B. bei Smart 1961), sondern sie drückt die enorme Bedeutung aus, die eine anständige Behandlung durch unsere Mitmenschen für uns persönlich hat. Es wohnt mit anderen Worten dieser Praxis ein emotionaler Kern inne, der unsere eigene Bindung an moralische Maßstäbe und unseren eigenen Platz in der Moralgemeinschaft reflektiert. Strawsons Betonung moralischer Gefühle und zwischenmenschlicher Beziehungen verknüpft Wallace sodann mit einem zweiten, eher kantianischen Gedankenstrang, indem er hervorhebt, wie wichtig Gerechtigkeit für die Praxis des Zur-Verantwortung-Ziehens ist, und ihren Zusammenhang mit rationalen Fähigkeiten aufzeigt: „Es ist gerecht, Menschen moralisch zur Verantwortung zu ziehen, wenn sie über das rationale Vermögen gebieten, moralische Gründe einzusehen und anzuwenden und ihr Verhalten im Lichte dieser Gründe zu steuern“ (Wallace 1994, S. 1). Anders ausgedrückt: „Das ,Können“, auf das es für die moralische Verantwortung ankommt, ist nicht das ,Können“ alternativer Möglichkeiten ..., sondern das ,Können“ eines allgemeinen rationalen Vermögens“ (Wallace 1994, S. 7 f.). Aus naheliegenden Gründen konzentriere ich mich auf diesen letzteren Strang.

Die Frage der Gerechtigkeit steht bei Wallace deshalb so im Vordergrund, weil sie das Hauptmotiv für den Verantwortungs-Inkompatibilismus bildet. Dessen Grundgedanke ist bekanntlich dieser: Wenn jemand determiniert ist, in einer bestimmten Weise zu denken oder zu handeln, scheint es nicht gerecht, ihn dafür in einer Form zur Verantwortung zu ziehen, die ihm Schaden oder Schmerz zufügt; denn mangels Verhaltensalternativen hat er keine Möglichkeit, solch widrige Reaktionen zu vermeiden. Diesem Gedanken will Wallace Genüge tun, ohne zuzulassen, dass er die Praxis des Zur-Verantwortung-Ziehens grundsätzlich in Frage stellt. Dazu will der Autor erstens die ,Möglichkeit, Tadel und Übelnehmen zu vermeiden', nicht ebenso metaphysisch wie mysteriös als ,Fähigkeit, anders zu handeln“ verstehen, sondern im geläufigeren Sinne eines rationalen Vermögens. Das ,Vermögen [. . . ], moralische Gründe einzusehen und anzuwenden“, ist eine normale, alltägliche Fähigkeit. Natürlich gelingt es den meisten Erwachsenen nicht immer, in der moralisch geforderten Weise zu handeln und zu urteilen - aber solange es ihnen regelmäßig gelingt, können wir ihnen jene Fähigkeit zuschreiben. Zweitens erläutert Wallace, wie Entschuldigungen und Befreiungen zu verstehen sind - Fälle also, in denen wir andere Menschen üblicherweise nicht verantwortlich machen für ihr Verhalten, auch wenn es in mancher Hinsicht falsch oder schädlich sein mag.

Entschuldigungen und Befreiungen sind unter anderem deshalb von Belang, weil das inkompatibilistische Standardargument so interpretiert werden kann, dass wir bezüglich unserer Missetaten allesamt entschuldigt sind, da wir in Anbetracht unserer Geschichte, Veranlagung und näheren Umstände gar nicht anders hätten handeln können - oder auch so, dass wir von moralischen Ansprüchen alle miteinander befreit sind, weil wir die Fähigkeit zum moralischen Handeln eigentlich gar nicht haben und es unter den Bedingungen des Determinismus sozusagen einen glücklichen Zufall darstellt, wenn tatsächlich einmal jemand rechtschaffen handelt. Wallace zeigt überzeugend, dass Entschuldigungen und Befreiungen nicht nach dieser Lesart funktionieren.

Wird nämlich jemand von moralischen Ansprüchen befreit, dann nicht deshalb, weil wir sein Handeln für determiniert halten würden, sondern weil es sich irgendwie 
seiner rationalen Kontrolle entzog: Einen solchen Menschen zur Verantwortung zu ziehen, wäre nach Wallace ungerecht oder, genauer gesagt, unvernünftig. Der Kleptomane beispielshalber unterliegt - im Gegensatz etwa zu jemandem, der um des persönlichen Vorteils willen ein Versprechen bricht - einem Zwang. Im noch gravierenderen Fall des Psychopathen fehlt es überhaupt an der Fähigkeit zur rationalen Selbstkontrolle, so dass es nachgerade sinnlos wird, ihn zur Verantwortung zu ziehen. Der Preis der Unfähigkeit des Psychopathen ist dabei keineswegs eine (ihm selbst mutmaßlich gar nicht unwillkommene) Befreiung von den Folgekosten der Verantwortungszuschreibung, sondern sein Ausschluss aus der moralischen Gemeinschaft. Man nimmt ihm gegenüber eine (wie Strawson es nennt) „objektive Einstellung“ ein, d. h. man begegnet ihm als Gefahr, als jemandem, der in Schranken zu halten und eventuell ärztlich zu behandeln ist. Auch die diversen Zwangsstörungen müssen, wenngleich auf niedrigerem Niveau, in Schranken gehalten, behandelt oder sonstwie bearbeitet werden; denn wiewohl wir eine Zwangsgestörte nicht tadeln, so erwarten wir doch, dass sie ihr Leiden erkennt und gegebenenfalls Hilfe annimmt, um es zu heilen oder in seinen Auswirkungen zu mildern.

Entschuldigungen wirken vergleichsweise unmittelbarer und punktueller. Wir entschuldigen jemandes Verhalten, wie schädlich oder unrecht es uns auch erscheinen mag, dann, wenn wir davon ausgehen, dass es nicht beabsichtigt war. Dass ich eine wertvolle Vase fallenlasse, wird man mir nachsehen, wenn ich keine andere Möglichkeit hatte, zu verhindern, dass ein in Ohnmacht Sinkender mit dem Kopf am Kaminsims aufschlägt. Wenn eine von Sorgen niedergedrückte Kollegin es verabsäumt, uns zu grüßen, machen wir uns klar, dass sie das nicht persönlich meint, und entschuldigen sie bereitwillig. Auch hier geht es nicht um die Abwesenheit anderer Verhaltensmöglichkeiten in irgendeinem metaphysischen Sinne, sondern um die Abwesenheit einer bösen Absicht. Entschuldigungen sind für Wallace eine Forderung der Gerechtigkeit: dessen, dass der Betreffende Tadel oder Strafe nicht verdient hat. Entweder hat er ohnehin nicht unrecht gehandelt, oder es wäre angesichts der Anforderungen und Belastungen, unter denen er stand, nicht gerecht, ihm sein Handeln, auf bestimmte Pflichten pochend, vorzuhalten.

Beiläufig sei hier ein Problem von Wallaces Argumentation erwähnt. Es versteht sich nämlich nicht von selbst, dass Gerechtigkeit die ,Leitnorm‘ für die Praxis des Zur-Verantwortung-Ziehens ist (Benson 1996). Auch die Achtung für Andere oder eine Wertschätzung moralischer Gemeinschaft ließe sich zur Begründung dieser Praxis anführen - womit Wallace stellenweise durchaus konform geht (z. B. Wallace 1994, S. 69, 234). Des Weiteren könnte man es (mit Pamela Hieronymi: siehe unten, Abschn. 7) für einen Fehler halten, Tadel als eine Art Benachteiligung oder Sanktion anzusehen, deren Verteilung die Frage der Gerechtigkeit überhaupt erst aufwirft. Indes stellt diese Frage, wie gesagt, ein häufiges Motiv für inkompatibilistische Positionen dar, weshalb der Nachdruck, den Wallace darauf legt, verständlich ist, auch wenn durch diese Akzentuierung weitergehende Fragen nach Wesen und Begründung der Praxis der Verantwortungszuschreibung offenbleiben.

Die Gerechtigkeitsthematik weist allerdings auf einen grundlegenden Aspekt hin. Wallaces Ausgangsfrage lautet, ob bestimmte Formen, in denen Menschen einander behandeln, gerecht seien. Im Gegensatz zu vielen anderen Autoren setzt er also keine 
metaphysische Tatsache betreffs zurechnungsfähiger Erwachsener zur Begründung der moralischen Verantwortungspraxis voraus. Zwar sind Tatsachen über Menschen offenkundig relevant für ihn: im Hinblick auf Befreiungen die Frage, ob jemandem die Fähigkeit, moralische Gründe einzusehen und anzuwenden, zukomme; im Hinblick auf Entschuldigungen die Frage, ob das äußere Verhalten eines moralisch kompetenten Menschen eine unrechte Absicht oder Einstellung widerspiegele. Zum Ausgangspunkt nimmt er sich aber eben die Überlegung, wie wir einander behandeln sollten, sowie die Hypothese, dass die Praxis des Zur-VerantwortungZiehens (bzw. des Entschuldigens oder Befreiens von Ansprüchen) in diesem Lichte zu begründen sei. Obgleich er es nicht genau so formuliert, macht er damit auf eine versteckte Ungereimtheit in der Argumentation des moralischen Inkompatibilismus aufmerksam: Diesem zufolge scheinen wir geradezu moralisch verpflichtet zu sein, Menschen für ihre Handlungen nicht verantwortlich zu machen, da jede unmoralische Handlung nur einen weiteren Beleg für die Unfähigkeit ihres Urhebers abgibt, anders zu handeln, als er es tatsächlich tut. Was will der Inkompatibilist dann aber jenen entgegnen, die gleichwohl fortfahren, andere für ihr Tun und Lassen zur Verantwortung $\mathrm{zu}$ ziehen? Es ist $\mathrm{zu}$ vermuten, dass seine Entgegnung kritisch ausfallen wird - womit sich die Aufgabe stellt, zu erklären, inwiefern diese Kritik sich vom moralischen Tadel unterscheidet. (In diesem Zusammenhang fällt auf, wie wenig in der Verantwortungsdebatte bisher über das Wesen des Tadels gesagt worden ist - ein Versäumnis, das aufzuholen man erst jüngst begonnen hat: vgl. z. B. Sher 2005; Coates und Tognazzini 2012. Auf diesen Punkt komme ich weiter unten in Abschn. 7 noch zurück.)

Damit soll nicht behauptet werden, Wallaces normativer Ansatz sei über jeden Zweifel erhaben oder stelle gar die einzig denkbare Form dar, unsere Praxis des ZurVerantwortung-Ziehens in eine Beziehung zu allgemeineren moralischen Normen zu setzen. (Neben dem am Rande bereits erwähnten kruden Utilitarismus wären hier noch die kontraktualistischen Ansätze eines Scanlon 1998 oder Lenman 2006 sowie die kantianische Sichtweise bei Ripstein 2004 zu nennen. Auf ihre Weise plädieren sie alle dafür, die Praxis des Zur-Verantwortung-Ziehens zum Beziehungstyp der fairen Interaktionen zu zählen.) Kritiker könnten aber vor allem versucht sein, beim Begriff des Vermögens oder Könnens anzusetzen und auf Klärung zu dringen.

So ist von inkompatibilistischer Seite vorgebracht worden, dass es für die Begründung der Praxis der Verantwortungszuschreibung nicht ausreiche, unser rationales Vermögen anzuführen, wenn jemandem bei einer bestimmten Gelegenheit die ,Fähigkeit‘ zur Ausübung besagten Vermögens fehlte. (Dieser Unterschied zwischen allgemeinem Vermögen und konkreter Fähigkeit findet sich mehrfach bei Wallace 1994: z. B. 182 f., 192, 199 ff.) Der Einwand ist leicht nachvollziehbar: Hat jemand sein allgemeines Vermögen nicht ausgeübt, dann war er vermutlich kausal determiniert, es nicht auszuüben; und somit taugt der Verweis auf das allgemeine Vermögen nicht zur Vindizierung einer konkreten ,Fähigkeit, anders zu handeln“ (vgl. etwa Cullity 1997; Kane 2002). Wallace versucht, diesen Einwand zu unterlaufen (Wallace 1994, S. 182 ff.), indem er seinen Grundgedanken zur Befreiung von der Verantwortung wiederholt, wonach die Befreiung unter Bezugnahme nicht auf kausale Determination, sondern auf eine Beeinträchtigung des Vermögens der 
rationalen Selbststeuerung gewährt wird. Darüber hinaus legt er dar, dass die auf praktische Aufgaben bezogene Modalbegrifflichkeit (wie die Kraft zum Heben schwerer Gewichte oder das Beherrschen einer zweiten Sprache) eine gänzlich andere sei als diejenige, die wir in Aussagen der Art verwenden, dass etwas, weil determiniert, nicht anders habe geschehen können (in Erwiderung auf Kane: vgl. Wallace 2002, S. 717 ff.).

An beiden Antworten mag man ein gewisses Ungenügen empfinden. Die erste sieht so aus, als übte Wallace einfach hinhaltenden Widerstand, anstatt sich genauer darüber auszulassen, wie rationales Vermögen und Verantwortungspraxis aufeinander zu beziehen wären. Gelegentlich deutet er zwar einen tieferen Zusammenhang an (z. B. Wallace 1994, S. 234), doch seine Betonung von Entschuldigungen und Befreiungen verleitet ihn m. E. zu einer Unterbewertung der Rolle, die wir - sofern nicht gerade entschuldigt oder befreit - bei der Aufrechterhaltung der Moralgemeinschaft als Teilnehmer spielen. Und während die zweite Antwort dem Problem so, wie es bei Kane gefasst ist, gerecht werden mag, bietet sie keine Lösung für die tiefergehende Frage an, welche Art von Modalkategorien für das Verständnis moralischer Handlungsfähigkeit denn gefordert sei. Gewiss tadeln wir andere manchmal dafür, nicht genügend Entschlossenheit oder Willenskraft an den Tag zu legen - die einschlägigen Modalkategorien für praktische Aufgaben -, aber das ist nicht unbedingt der typische Fall. Insbesondere geht es bei schlechten Menschen nicht einfach nur darum, dass sie sich größere Mühe geben müssten, gut zu sein (oder zu handeln), und Gutsein ist schwerlich dem Anspannen eines Muskels vergleichbar. Würden wir die Dinge so betrachten, wäre der Schluss nicht weit, dass bei manchen Menschen der ,moralische Muskel' schlechterdings, wieviel sie ihn auch immer bewegen oder trainieren, schlaffer ist als bei anderen - womit die Sorge um die Gerechtigkeit der Verantwortungszuschreibung unweigerlich wieder Einzug hielte. (Tatsächlich scheint Wallace auf den letzten Seiten seines Buches in diese Richtung zu tendieren, wenn er beschreibt, wie eine mangelhafte Erziehung und schlechte soziale Verhältnisse das Vermögen der rationalen Selbstkontrolle beeinträchtigen; Wallace 1994, S. 231 ff.) Im Übrigen gesteht Wallace freimütig, dass er „den Begriff eines allgemeinen Vermögens weitgehend als unproblematisch voraussetze“ (Wallace 1994, S. 192). Auch wer ihm darin folgt, dass das Nachdenken über Verantwortung nicht von einer Metaphysik des rationalen Handlungsvermögens ausgehen sollte, wird der Ansicht sein, dass es über unser moralisches und rationales Vermögen ein bisschen mehr zu sagen gibt.

\section{Fischer und Ravizza, Responsibility and Control: $A$ Theory of Moral Responsibility (1998)}

Als Wallaces Buch erschien, hatte John Martin Fischer bereits begonnen, eine Auffassung von moralischer Verantwortung zu entwickeln, die unsere Fähigkeit zur moralischen Reflexion betont (Fischer 1982, 1987, 1994). Am umfassendsten findet sie sich in Fischer und Ravizza dargelegt (Fischer und Ravizza 1998), einem viel diskutierten Werk, das hier als der avancierteste Ansatz in puncto 
,Empfänglichkeit für Gründe` vorgestellt wird. In ihm schneiden die Autoren wichtige Themen an, insbesondere mit ihrem Hinweis auf die Bedeutung der Übernahme von Verantwortung für uns selbst. Ihre Konzeption ähnelt in vielerlei Hinsicht der Wallaces, nimmt sich aber auch der von diesem offengelassenen Frage an, wie unser moralisches Denken und Urteilen zu verstehen sei. Dennoch bleibt die Fassung der Problematik bei Fischer und Ravizza, wie ich zeigen werde, hinter ihren Möglichkeiten zurück.

In Grundzügen entfaltet sich die Theorie folgendermaßen: Welche Art von Kontrolle üben verantwortliche Personen über ihre Handlungen aus? Wie zahlreiche Kompatibilisten dargetan haben, ist es unwahrscheinlich, dass wir irgendwie zwischen alternativen Möglichkeiten wählen können, jedenfalls in einem starken metaphysischen Sinne. Wohl aber denken wir zuweilen über verschiedene Handlungsoptionen nach und erwägen ihr Für und Wider - mit anderen Worten, wir lassen uns in unserem Handeln von Gründen leiten. Fischer und Ravizza meinen nun, dass für moralische Verantwortung diese Art von „Leitkontrolle“ (,guidance control“) hinreichend sei. Die Leitkontrolle - da uns aus der Erfahrung wohlvertraut - erlaube es zudem, systematische Fragen wie die nach ihrer kausalen Grundlage sowie danach, ob diese Grundlage deterministisch zu interpretieren sei, unentschieden zu lassen. Fischer und Ravizza nennen ihre Theorie „semi-kompatibilistisch“, da sie den Determinismus weder als wahr noch als falsch unterstellt.

Das Hauptargument unserer beiden Autoren dafür, dass moralische Verantwortung keine „Weichenstellungskontrolle“ (,,regulative control“) über „alternative Möglichkeiten" verlangt, stützt sich auf Szenarien, in denen jemand nur in einer ganz bestimmten Weise handeln kann - nicht aus eigener Wahl, sondern weil zum Beispiel ein Manipulator ihn dazu bringt: ein verrückter Wissenschaftler etwa, der von außen Einfluss auf das Feuern seiner Neuronen nehmen kann - und dennoch verantwortlich ist. Auch dieser Diskussionsstrang verdankt einiges Harry Frankfurt (Frankfurt 1969), dem vornehmlichen Schöpfer solcher Fantasien. Es sind aber auch prosaischere Beispiele denkbar, in denen einem Handelnden ohne sein Wissen die Möglichkeit, anders zu handeln, gewissermaßen schon materiell verbaut ist. Fischer und Ravizza stellen uns die Situation eines nach rechts abbiegenden Autofahrers vor Augen, dem nicht bewusst ist, dass sein Wagen aufgrund externer Faktoren gar nicht anders fahren kann (Fischer und Ravizza 1998, S. 32; ebenso Fischer 2012, S. 120 f.). So konstruiert das Beispiel auch wirkt, so verdeutlicht es doch den Sinn, in dem man sagen könnte, der Fahrer lenke die Bewegung seines Fahrzeugs und sei demzufolge verantwortlich für das Rechtsabbiegen, obwohl er faktisch in gar keine andere Richtung hätte steuern können. Dieser Argumentationsmethode - man könnte sie ,Intuitionsantrieb durch verstiegene Beispiele' nennen - kommt natürlich keine begründende Kraft zu, da Beispiele wie Intuition gleichermaßen anfechtbar sind. Das Argument, so wäre also zu ergänzen, ist mitnichten unentbehrlich für die Theorie zumal Fischer (Fischer 2012, S. 123) die Gültigkeit anderweitiger Argumente (etwa der von Strawson vorgebrachten) gegen die Notwendigkeit der Weichenstellungskontrolle anerkennt.

Auf einem anderen Blatt steht die Behauptung, das Vorliegen von „Leitkontrolle“ sei hinreichend für moralische Verantwortung. Fischer und Ravizza weisen hier 
darauf hin, dass verschiedene kausale Pfade zu einer Handlung führen können, von denen manche der Rede von Verantwortung zweifellos den Boden entziehen: Handele ich aufgrund von hypnotischer Beeinflussung oder weil mir ein Betäubungsmittel ins Getränk gemischt worden ist oder auch infolge abseitiger Manipulationen eines bösen Wissenschaftlers, so bin ich nicht verantwortlich. Die positive Aussage der Theorie ist demnach die, dass es einen kausalen Pfad gibt, der moralische Verantwortung zulässt: Die Handlung muss aus einer Art von „Mechanismus“ hervorgehen, der erstens empfänglich für Gründe ist und zweitens dem Handelnden selbst zugehört.

Auf beide Bedingungen kommt es an, und keine der beiden hängt sei's von der Frage der Wahrheit oder Falschheit des Determinismus, sei's von den spezifischen Kausalpfaden ab, die geistigen Vorgängen und körperlichen Bewegungen zugrunde liegen. In Bezug auf die erste Bedingung erklären Fischer und Ravizza, der fragliche Mechanismus - beispielsweise eine „gewöhnliche praktische Überlegung“ oder „Abwägung aufgrund eines schwer widerstehlichen Wunsches" (Fischer und Ravizza 1998, S. 48) - müsse nicht hundertprozentig zuverlässig funktionieren. Sprächen wir immer angemessen auf Gründe an, so gäbe es vermutlich gar keine Notwendigkeit, einander zur Verantwortung zu ziehen. Über eine gewisse ,Robustheit' sollte der Mechanismus jedoch verfügen: Verantwortlich nennen wir nicht jemanden, der nur in einigen wenigen, speziellen Hinsichten oder in Ausnahmesituationen auf Gründe anspricht. Diese Forderung entspricht offenbar ziemlich genau Wallaces Rede von einem Vermögen, Gründe einzusehen und anzuwenden: Bei einer verantwortlichen Person zeigt sich dieses Vermögen weder unfehlbar noch bloß sporadisch.

Mit dem Vorliegen eines halbwegs zuverlässigen „Mechanismus“ ist es indes noch nicht getan. Fischer und Ravizza ersinnen Fälle, in denen jemand infolge der Machenschaften eines (nicht-bösen?) Neurowissenschaftlers, einer Gehirnwäsche oder Hypnose ebenfalls halbwegs zuverlässig auf Gründe anspricht. Die Methode des ,Intuitionsantriebs durch verstiegene Beispiele' einmal zugestanden, leuchtet es ein, dass der Manipulierte selbst nicht als verantwortlich Handelnder gelten kann. Sowohl das Nachdenken über Gründe als auch die Verantwortung liegen beim Strippenzieher. Der Mechanismus, der auf Gründe anspricht, muss der des Handelnden selbst sein.

Was macht einen Mechanismus zu dem eines Handelnden? Für Fischer und Ravizza erfordert das einen Entwicklungsprozess, aufgrund dessen der Betreffende dahin gelangt, sich (i) als Ursprung seiner Entscheidungen und Handlungen und (ii) als für diese verantwortlich anzusehen - zum Beispiel, indem er akzeptiert, dass andere, sollte er unrecht gegen sie handeln, ihm das mit gutem Grunde übelnehmen könnten. Im Regelfall vollzieht sich diese Akzeptanz stillschweigend im Laufe des Heranwachsens und der Sozialisation innerhalb einer Gemeinschaft anständiger Personen. Dabei übernimmt der Mensch allmählich „die Verantwortung für die Quellen seines Handelns [und] eignet sie sich in einem wichtigen Sinne an" (Fischer und Ravizza 1998, S. 210). Wer sich selbst nicht als Ursprung seines eigenen Handelns sieht, wird nach Fischer und Ravizza „vermutlich nicht den Versuch unternehmen, mittels Entscheidungen oder Körperbewegungen auf die Welt 
einzuwirken" (Fischer und Ravizza, S. 218). Und wer sich nicht als verantwortlich begreift, schneidet sich selbst von normalen menschlichen Beziehungen ab und verliert sogar seine personale Einheit. Die Autoren geben das Beispiel eines Serienmörders, der seine Verbrechen einem ,inneren Monster“ - der für Gründe nichtempfängliche Mechanismus par excellence! - zur Last legt (Fischer und Ravizza, S. 219 f.).

All das sind wichtige Gesichtspunkte. Verschiedene „Mechanismen“ zu bemühen, hat den Vorteil, berücksichtigen zu können, dass ein und derselbe Mensch für sein Tun manchmal verantwortlich ist und manchmal nicht. Er ist es, wenn seine Handlungen dem Mechanismus ,gewöhnliche praktische Überlegung“ entspringen (Fischer und Ravizza, S. 216); er ist es nicht, wenn seine Taten durch einen der Vernunft unzugänglichen, möglicherweise auf einem inneren Zwang, einer Sucht oder geistigen Störung beruhenden Mechanismus zustande kommen. Fruchtbar ist auch die Idee, dass Verantwortung entsteht, indem wir Verantwortung für unser Handeln übernehmen, und dass die Übernahme von Verantwortung für eine vollständige Teilhabe an normalen menschlichen Beziehungen wesentlich ist. Auf diese letzteren beiden Punkte, bei denen die Autoren zu meinem Bedauern nur kurz verweilen, komme ich noch zurück.

Neben diesen Vorzügen sind auch einige Schwierigkeiten zu vermerken, die sich aus der Darstellung ergeben. Das menschliche Denk- und Urteilsvermögen als „Empfänglichkeit für Gründe“ auszulegen, bringt schon einmal die Gefahr mit sich, es allzu passiv zu konzipieren. Diese Empfänglichkeit dann aber ihrerseits als „Mechanismus“ zu fassen, ist ein noch weit radikalerer Schritt, der etwas, das wir tun, vollends umdeutet zu etwas, das geschieht - entweder in uns oder, bei den exzentrischeren Beispielen von Manipulation, außer uns (vgl. jedoch Fischer et al. 2007, S. 78). Damit tut sich eine skeptische Lücke auf; denn wie kann dieses Geschehen jemandes Tun sein? (En passant sei gesagt, dass ebendiese Art von Lücke dem Inkompatibilismus in die Hände spielt. Wenn wir menschliche Tätigkeit quasi mechanistisch, im Sinne von Kausalprozessen beschreiben, wird es schwer, ein handelndes Subjekt namhaft zu machen, das irgendetwas tut.) In der Anwendung auf Denk- und Urteilsprozesse sind mechanistische Bilder klarerweise fehl am Platze: Es gibt kein Denken ohne ein Subjekt, das denkt. Anders gesagt, die Idee der Verantwortungsübernahme dient hier dazu, ein künstliches Problem zu lösen. Überdies erzeugt sie einen logischen Zirkel: Jemandes geistige Tätigkeit - d. h. ein (wie auch immer unsprachlicher oder unausgesprochener) Denk- und Urteilsprozess, mithin, gemäß Fischer und Ravizza: die Aktivität eines Mechanismus - wird ins Feld geführt, um zu erklären, in welcher Weise die Aktivität eines Mechanismus das verantwortliche Handeln von jemandem repräsentiert.

Nun ist solche Wirrnis nicht rein artifiziell, auch wenn eine undurchdachte ,Mechanismus'-Metapher sowie eine Vorliebe für weit hergeholte Beispiele sie unnötig vermehren. Letztlich verweist sie auf die Grundfrage, wie die Fähigkeit zum rationalen und verantwortlichen Handeln - und mit ihr der einschlägige Begriff der Fähigkeit selbst - eigentlich zu verstehen sei.

Diese Grundfrage soll in den folgenden Abschnitten über Angela Smith und Pamela Hieronymi wieder aufgenommen werden. Zuvor lohnt es sich, noch einen 
näheren Blick auf den Begriff der Verantwortungsübernahme zu werfen. Fischers und Ravizzas Konstruktion mag etwas forciert sein, die aufgezeigte Zirkularität aber, soviel kann man ihnen zugutehalten, ist dem Gegenstande inhärent. Keiner von uns hat sein eigenes Handlungsvermögen hervorgebracht; jeder von uns ist ein Produkt sozialer und biologischer Faktoren, über die er zwangsläufig nicht verfügt; jeder von uns ist endlich und fehlbar: Und doch müssen wir, wenn Moral und Rationalität denn wirksame Kräfte in der Welt sein sollen, alle danach streben, beider Ansprüche unserem Handlungsvermögen zu inkorporieren.

Verantwortungsübernahme in diesem Sinne hängt teilweise von der Entscheidung ab, sich an gültige Normen zu halten. Wo das geschieht, gibt es keinen guten Grund, jemanden zur Verantwortung zu ziehen, da er innerlich bereits selbst die Verantwortung übernommen hat. Verantwortungsübernahme hat aber auch ein retrospektives Moment, das von der Bereitschaft lebt, sich selber zur Verantwortung zu ziehen, berechtigte Vorwürfe zu akzeptieren und aus ihnen zu lernen. Zu dieser Bereitschaft muss, soll sie keine leere Floskel bleiben, eine Scheu davor gehören, Ausflüchte zu suchen oder den Schwarzen Peter weiterzureichen - eine in unserem Zusammenhang besonders wichtige Abneigung also gegen jedes Sich-Herausreden, von wegen man habe nicht anders handeln können, weil man durch Biologie und Eltern und Gesellschaft nun einmal so geworden sei. Unabhängig davon, wie wir im Einzelnen die Geschichte verstehen, durch die wir geworden sind, wer wir sind, dürfen wir unser Handeln nicht auf ein Produkt wirkender Kausalkräfte reduzieren. Das würde, wie wir von Wallace wissen, dem Sinn des Entschuldigens selbst zuwiderlaufen. Entschuldigungen zeigen an, dass wir, auch wenn der Anschein gerade dagegen spricht, nicht auf eine bestimmte, unrechte Denk- oder Handlungsweise festgelegt sind - sie zeigen nicht an, dass wir außerstande wären, uns selbst auf eine Handlungsweise festzulegen. Und wie Robert Adams in einem berühmten Aufsatz festgestellt hat: „Die Übernahme der Verantwortung für die eigenen Gefühle und Motive abzulehnen, hieße [erstens], sich dem eigenen Empfindungs- und Begehrungsvermögen in unangemessener Weise zu entfremden ", und zweitens, eine entscheidende Gelegenheit zum moralischen Lernen auszuschlagen (Adams 1985, S. 16).

Verantwortungsübernahme ist also ein fundamentaler Bestandteil der Fähigkeit zu verantwortlichem Handeln. Weiter oben ist in Auseinandersetzung mit der Problematik des Fähigkeitsbegriffs bei Wolf bereits angedeutet worden, dass es einen konstitutiven Zusammenhang zwischen der Fähigkeit zu verantwortlichem Handeln und der Praxis des Zur-Verantwortung-Ziehens geben könnte. Hier ist nun die Pointe, dass die Norm der Verantwortungsübernahme - die Norm, die uns dazu auffordert, uns selbst zur Verantwortung zu ziehen, wie man auch sagen könnte - die Fähigkeit zu verantwortlichem Handeln mitkonstituiert und damit zur Sinnhaftigkeit unserer Praxis der wechselseitigen Verantwortungszurechnung beiträgt. Dass zum Erwachsenwerden typischerweise die Übernahme der Verantwortung für das eigene Tun, Lassen und Selbst gehört, ist mehr als eine soziologische Beobachtung: Es ist eine unerlässliche Pflicht. Wir müssen lernen, unser eigenes Handeln zu reflektieren und im Lichte normativer Ansprüche zu bewerten sowie zu akzeptieren, dass andere Menschen mitunter befähigt und berechtigt sind, unsere Bewertungen zu korrigieren. Fischer und Ravizza können plausibel darlegen, dass jemand, der partout keine 
Verantwortung für sich übernimmt, schlicht der Fähigkeit ermangelt, für Gründe empfänglich zu sein, und ergo nicht verantwortungsfähig ist. Diese These verweist jedoch auf einen grundsätzlicheren Punkt. Denn was immer sonst über die Gründe zu sagen sein mag, für die verantwortungsfähige Personen empfänglich sein sollen, eines ist gewiss: Diese Gründe müssen auch Gründe dafür einschließen, die Verantwortung für sich selbst zu übernehmen, d. h. sich selbst als aktiven und zurechnungsfähigen Träger von Bindungen und Verpflichtungen zu verstehen.

\section{$6 \quad$ Angela M. Smith: Verantwortung liegt im Urteil, nicht in der Wahl}

Angela Smith und Pamela Hieronymi sind einer der schwierigsten Fragen überhaupt in Bezug auf die Fähigkeit, rational und verantwortlich zu handeln, nachgegangen und haben dabei vielfach ähnliche Positionen entwickelt. Die Frage geht auf das Grundproblem zurück, vor dem Kompatibilisten und Inkompatibilisten gleichermaßen stehen: Wie können wir das Handlungsvermögen so ansetzen, dass einerseits seine Beziehung zu biologischen und sozialen Prozessen erkennbar wird, so auch, dass wir empirisch beobachtbare Ereignisse, wie sie zu einer kausal wohlgeordneten Welt gehören, aus ihm hervorgehen sehen - und so, dass es andererseits diesen Ereignissen weit genug entrückt ist, um eine unabhängige, zurechnungsfähige Quelle von Aktivität bilden zu können? Oder, im Rekurs auf die von Fischers und Ravizzas ,Mechanismus'-Metapher gestiftete Verwirrung formuliert: Wie können wir in einer Welt des Geschehens Platz fürs Tun schaffen? Die inkompatibilistische Antwort mystifiziert die Sache eher: Die famose „Fähigkeit, anders zu handeln“ etwa zeigt ein Problem an, ohne es zu lösen. Die oben dargestellten kompatibilistischen Ansätze wiederum stützen sich auf den Gedanken des rationalen Vermögens, d. h. der Fähigkeit, mit Bezug auf Gründe zu denken, zu urteilen und zu handeln - einer Fähigkeit, die, wie sehr sie uns auch praktisch vertraut sein mag, der philosophischen Aufklärung bedarf.

Eine Möglichkeit, etwas Halt in diesen Untiefen zu finden, besteht darin, das Augenmerk auf den Zusammenhang von Verantwortung und Kontrolle zu richten. Etliche Autoren haben jüngst im Anschluss an Adams (Adams 1985) darauf hingewiesen, dass der geläufige Gedanke, Personen seien für das verantwortlich, was sie kontrollierten, einen kleinen Haken hat: Er verfehlt nicht wenige signifikante Fälle der Zuschreibung von Verantwortung. So hat George Sher in seinem Aufsatz, Out of Control` (Sher 2005) in einprägsamer Weise Beispiele für unrechtes Handeln aufgrund von Fahrlässigkeit, Unachtsamkeit, Panik, voreiligem Urteil, Gefühllosigkeit und moralischem Irrtum vorgeführt. In keinem dieser Fälle scheint man mit Fug sagen zu können, der Betreffende habe sein Handeln bzw. Unterlassen in dem Sinne kontrolliert, dass er eine bewusste Wahl getroffen hätte, unrecht zu handeln; und doch liegt es jedes Mal klar am Tage, dass er für sein Tun oder Lassen Tadel verdient hat.

Schon zuvor hatte Angela Smith in ihrem Aufsatz ,Responsibility for Attitudes“ (Smith 2005) über Einstellungen nachgedacht, wie sie sich z. B. in Vergesslichkeit 
oder in spontanen Reaktionen, in Einfällen, die uns ,kommen', und in Zufällen der Wahrnehmung ausdrücken. Smiths Hauptthese geht dahin, dass die Aktivität, derentwegen wir einander und uns selbst zur Verantwortung ziehen, „nicht die der Wahl, sondern die der Wertung", des wertenden Urteilens ist (Smith 2005, S. 237). Spontane Reaktionen und gedankenlose Versäumnisse verraten nicht weniger über uns als unsere vorsätzlichen Handlungen, insofern sie zum Vorschein bringen, was uns wichtig ist und sinnvoll erscheint. Diese Wertung wird und muss im Allgemeinen nicht ausformuliert werden. Trotzdem wäre es falsch, sie als etwas Passives zu beschreiben. Sie charakterisiert einen Menschen - sie widerfährt ihm aber nicht.

Um nicht missverstanden zu werden: Smith ist wohlbewusst, dass manche mentalen Zustände wesensmäßig passiv sind. So bin ich einer Sinnesempfindung wie Schmerz oder Durst normalerweise einfach ausgeliefert. Sie enthält noch kein Urteil, keine Wertung, und sie wirft auch nicht die Frage nach der Verantwortung auf. (Verantwortungsfragen können sich allerdings an meiner Einstellung zu der betreffenden Empfindung entzünden: wenn ich beispielsweise die sich in ihr zeigenden körperlichen Bedürfnisse missachte oder wenn ich umgekehrt Dringlicheres ignoriere.) Sinnesempfindungen stehen höchstens in einer kausalen Relation zu unseren Einstellungen: Eine ängstliche Einstellung aufgrund des Urteils, dass Gefahr drohe, kann eine Empfindung wie Übelkeit verursachen; vernachlässige ich mich selbst, sind Hunger und Durst die absehbaren Folgen. Empfindungen können außerdem nur nützlich oder schädlich, angenehm oder unangenehm sein. Urteile hingegen unterliegen dem Maßstab von wahr und falsch, recht und unrecht, vernünftig oder unvernünftig; und ebenso die Einstellungen, in denen die Urteile sich manifestieren.

Smiths Grundidee ist also die, dass unsere Gedanken und Einstellungen, Handlungen und Unterlassungen die Haltung erkennen lassen, die wir zu allen möglichen normativen Fragen einnehmen: etwa zu der, was man den Freunden im Gegensatz zu Fremden schuldig sei, oder zu jener der Priorität verschiedener Angelegenheiten und Projekte. Wenn ich, um ein Beispiel von Smith zu verwenden, den Geburtstag eines Freundes vergesse, sagt das etwas über die Bedeutung dieses Tages innerhalb meines Koordinatensystems aus (dass er z. B. weniger wichtig ist als meine aktuelle Ehekrise oder, je nachdem, als meine neueste Affäre). Diese Einstellung kann gegebenenfalls natürlich falsch sein; aber der Fehler liegt dann nicht so sehr in einer Wahl als in einem Aufmerksamkeits- und Bindungsmuster. Das Muster offenbart meine Prioritäten und bringt meine Urteile zum Vorschein - wobei ,Urteil' in einem weiten Sinne zu verstehen ist als Einschätzung der Welt und meiner Position in ihr und weniger als spezifische Erkenntnis, die ich zu einem bestimmten Zeitpunkt habe (Smith 2005, S. 251 f.). In gewisser Weise ist nichts wesentlicher an einem Menschen als ebendiese Einschätzungen. Deshalb kann auch ein Akt des Vergessens tadelnswert sein oder zumindest eine Rechtfertigung erheischen, denn er steht, wie Smith sagt, in einer „rationalen Beziehung“ zu einem Werturteil - zu dem, was mir wichtig ist. Smith spricht manchmal (im Anschluss an Scanlon 1998, S. 18-22) von „urteilsbezogenen Einstellungen“, was aber nicht meint, dass unsere Einstellungen von unseren Urteilen verursacht würden. Bei einem vollkommen rationalen Subjekt, gesetzt, es existierte, würden die Urteile stets seinen Einstellungen, Reaktionen und so weiter entsprechen. Bei einem nur teilweise rationalen Subjekt, wie wir alle es 
sind, sollten die Einstellungen usw. den Urteilen entsprechen (Smith 2005, S. 253). Wenn ich, um erneut auf Smiths Beispiele zurückzugreifen, Hausspinnen für harmlos halte, sollte ich nicht mit Schrecken auf sie reagieren; wenn mein Freund mir wichtig ist, sollte ich, außer in Krisen und Notfällen, an seinen Geburtstag denken. Lob und Tadel sind folglich nach mehreren Dimensionen hin zu vergeben, nämlich danach, ob jemandes im Handeln sich manifestierende Einstellungen vernünftig sind, ob die zugrunde liegenden Werturteile vernünftig sind, und ob die Handlungen, Einstellungen und Werturteile miteinander übereinstimmen.

Hinter diesen Ausführungen steht die tiefere Frage nach unserem Verständnis der Aktivität verantwortungsfähiger Personen. „,[D]ie Scheidelinie zwischen Aktivität und Passivität ist das Urteil“", schreibt Smith (Smith 2005, S. 263). Das Distinktionsmerkmal des rational Handelnden besteht nicht in der Fähigkeit, als unverursachte Ursache zu handeln, sondern in der Fähigkeit, eine wertende Haltung einzunehmen und Gründe zu ihrer Rechtfertigung ebenso vorzubringen wie von anderen zu fordern. Im Alltagsleben findet dieses Geben und Fordern zumeist nicht in Gestalt einer gesitteten Debatte am Seminartisch statt - es kann ohne weiteres als wütende Anklage auftreten, dass eine Handlung oder Unterlassung nicht in Ordnung sei. Aus all dem folgt, dass die Metaphysik des verantwortlichen Handelns sich weniger mit Fragen von Kausalität und Determinismus als vielmehr mit den Distinktionsmerkmalen von Rationalität und Werturteil beschäftigen sollte. Insbesondere sollte sie das Handeln nicht als ein in einer bestimmten Weise verursachtes Ereignis darstellen, sondern eher als Manifestation eines rationalen Vermögens: des Vermögens, zu beurteilen, was wichtig ist, und danach zu handeln.

Wie alle an dieser Debatte beteiligten Autoren sieht auch Smith, dass Erziehung und Kultur einen prägenden Einfluss auf unsere Wertbindung sowie auf unsere Fähigkeit, diese zu revidieren, ausüben. Sie anerkennt also auch, dass sich weitergehende Fragen nach der Verantwortung stellen können, die jemand dafür trägt, der geworden zu sein, der er ist (Smith 2005, S. 267 f., 2008, S. 389 f.). Die jeweilige Antwort, so Smith, kann sich durchaus darauf auswirken, wie wir auf jemanden reagieren. Sie ändert aber nichts daran, dass ein Mensch auf seinem Weg durch die Welt eine bestimmte Wertbindung zum Ausdruck bringt und dass diese Bindung für seine Umgebung im Guten wie im Bösen von höchster Bedeutung ist. Ebenso wenig ändert sie etwas daran, dass die Wertbindung rational vertretbar sein sollte. Ungeachtet dessen außerdem, wie viele Zugeständnisse wir geneigt sind zu machen, sobald wir zu verstehen suchen, wie andere zu denen geworden sind, die sie sind, sollten wir vorsichtig damit sein, in ihnen ,passive Opfer ihrer Fehlurteile“ (Smith 2008, S. 390) oder gleich ihrer gesamten Erziehung und Lebensumstände zu sehen: Eine solche Haltung kann als geringschätzig oder respektlos empfunden werden, und - so ließe sich hinzufügen - sie ist auch nur schwer durchzuhalten, wenn man einem Menschen gegenübersteht, der keinen Anlass sieht, von seinem unvernünftigen oder unmoralischen Benehmen abzugehen.

In diesem Abschnitt standen Fälle von Verantwortung ohne Handlungskontrolle zur Debatte; denn dass spontane, unüberlegte Reaktionen moralisch nicht ins Gewicht fallen und keine moralisch relevanten Charakterzüge offenbaren würden, wäre eine äußerst unplausible Behauptung. Im Gegenteil ist die Schadenfreude, die ich 
über das Missgeschick eines Freundes empfinde, zu tadeln und tadelnswerter noch, wenn ich sie, wie unbeabsichtigt auch immer, zeige. Manche Philosophen, die von der Bedeutung der Kontrolle für die Zuschreibung von Verantwortung überzeugt sind, wenden ein, dass ich auf jeden Fall zu einem früheren Zeitpunkt eine entsprechende Wahl getroffen haben müsse (z. B. Otsuka 1998; Michael Smith 2004b; Levy 2005). Doch wenn wir uns daraufhin vorstellen, ein Mensch hätte aktiv beschlossen, unsensibel oder gemein zu werden, oder wäre, hätte er solches beschlossen, nurmehr für spontane Anwandlungen von Unsensibilität und Gemeinheit zu tadeln, dann sehen wir das Gewaltsame solcher Argumente, die sich nach vorgefassten theoretischen Konzepten statt nach den Realitäten menschlichen Agierens und Interagierens richten.

Eine grundsätzlichere Frage steckt hinter der Unplausibilität derartiger Versuche, die Kontrollbedingung für den Verantwortungsdiskurs zu retten - beziehungsweise hinter ihrer Plausibilität, wie sich auch sagen ließe, insofern man zu ihrer Verteidigung ja anführen kann, dass sie der Schwierigkeit Ausdruck verleihen, zu verstehen, wie eine Tätigkeit zugleich die unsere und nicht unter unserer Kontrolle sein kann. Um welche Art von Aktivität geht es also bei der Fähigkeit, rational und verantwortlich zu handeln, und ihrer Manifestation in oft unwillkürlichen Urteilen und Akten?

\section{Pamela Hieronymi: Verantwortliche Tätigkeit als "Wertungskontrolle"}

Zur Beantwortung dieser Frage wende ich mich nunmehr den Arbeiten Pamela Hieronymis zu. Hieronymis gehaltvolle Überlegungen zur Verantwortung stehen denen von Angela Smith sehr nahe, doch hat sie sich eingehender mit dem Wesen geistiger Tätigkeit befasst. Wenn wir diese Tätigkeit und was zu ihr gehört, sorgfältiger bedenken, dann, so lautet im Kern ihr Fazit, stellen wir fest, dass Kontrolle, bei aller ihr zukommenden Bedeutung, nicht die primäre Erscheinungsform der Fähigkeit zum verantwortlichen Handeln ist.

Gemäß Hieronymis Hauptargument (wozu auch Smith 2004a; Moran 2012; Boyle 2011, 2013) unterliegen weite Bereiche unseres geistigen Lebens nicht unserer Kontrolle und könnten das auch gar nicht, und zwar in folgendem Sinne: Ob wir etwas glauben, übelnehmen oder beabsichtigen, können wir nicht auf der Basis irgendwelcher alten Gründe entscheiden, welche die Aufrechterhaltung eines solchen Geisteszustandes als wünschenswert erscheinen lassen mögen. Um beim Glauben zu bleiben: Etwas richtig glauben kann ich nur aus Gründen, die für seine Wahrheit sprechen. Es macht mich zwar vielleicht glücklicher, zu glauben, ich sei beliebter, als ich es tatsächlich bin; allein, das ist nicht die Art Grund, die mich von meiner besonderen Popularität überzeugen kann oder jedenfalls sollte. (Unter Missachtung der Tatsachen zu der Überzeugung zu gelangen, ich sei beliebt, käme reinem Wunschdenken gleich.) Überzeugungen zielen, wie es häufig heißt, auf Wahrheit. Wir könnten auch sagen: Überzeugungen sind den Tatsachen verantwortlich. Doch wiewohl wir nicht ,nach Belieben' glauben können, ist das Glauben bzw. Unterhalten 
von Überzeugungen etwas, das wir tun (vgl. a. Korsgaard 2010). Und es ist etwas, wofür wir einstehen müssen: Wir sollten imstande sein, in gewissem Umfang Rechenschaft über die Gründe für unsere Überzeugungen abzulegen, und Andere können uns mit Recht für unbegründete Überzeugungen kritisieren, z. B. wenn wir uns dem Wunschdenken hingeben.

Überzeugungen sind aber, wie gesagt, nur ein Beispiel. Unsere gesamte geistige Tätigkeit unterliegt der von Hieronymi so genannten „Wertungskontrolle“ (,evaluative control“) - unsere Absichten, Gefühle von Dankbarkeit, Übelnehmen oder Wohlwollen, die wir anderen entgegenbringen, usw. (Hieronymi 2006). Sie alle kommen nicht nach unserem Belieben zustande, sondern als Reaktion auf die Welt und als unsere Auffassung dessen, was der Mühe wert und lohnend in ihr ist. Ohne diesen Hintergrund würde keine Entscheidung uns vernünftig oder sinnvoll erscheinen. Unsere vorsätzlichen Handlungen legen von dieser wertenden Tätigkeit nicht weniger Zeugnis ab als unsere spontanen Reaktionen, und auch dem, was zu tun wir unterlassen oder vergessen, liegt sie vielfach zugrunde.

Es gibt noch eine weitere Hinsicht, in der unsere Handlungskontrolle begrenzt ist: Wir können uns nicht einfach entscheiden, mit unserem Verstehen und Verhalten richtigzuliegen. Im Falle der Überzeugung kann ich zum Beispiel auf mehr oder minder zuverlässige Überprüfungsmethoden zurückgreifen und bekannte Fehlerquellen meiden. Eine Garantie bieten mir solche Vorsichtsmaßnahmen aber nicht, und ich kann auch nur gelegentlich Gebrauch von ihnen machen. Dasselbe gilt in hohem Maße vom Urteil - und betrifft damit einen der für diesen Beitrag einschlägigen Grundbegriffe -, unserer Einschätzung also von Personen und Situationen. Solche Urteile hängen in mannigfacher Weise mit unseren Gefühlen und Gewohnheiten zusammen und üben gemeinsam mit ihnen einen gewaltigen Einfluss auf unseren Umgang mit Anderen aus. Zugleich sind sie äußerst fehlbar. Denn wieviel Mühe wir uns auch mit der kritischen Prüfung unserer Vorurteile, Annahmen oder gefühlsmäßigen Reflexe immer geben mögen, wir können doch nie aus uns heraustreten, um unsere Deutungen nachhaltig zu revidieren. Seine geistige Verfassung kann niemand hinter sich lassen. ,Kontrolle', soweit wir darüber verfügen, kann einzig im Rahmen der Fähigkeiten, die uns schon eignen, und der Perspektive, die wir bereits einnehmen, ausgeübt werden.

In einem wichtigen Betracht ist diese letztere Behauptung allerdings etwas voreilig. Wenn wir nämlich Glück haben, können wir von anderen Menschen, mit denen wir die Welt teilen, lernen und aus ihrer Reflexions- und Urteilsfähigkeit Nutzen ziehen. Dies gemahnt uns an einen Gesichtspunkt, der bereits im Zusammenhang mit Wolfs Fähigkeitsbegriff ins Spiel kam: Der soziale Kontext kann unsere Fähigkeit, ein Urteil zu revidieren, stärken oder schwächen. Wenn wir (moralisch) begünstigt sind, dann finden sich Menschen in unserer Umgebung bereit, uns unsere Versäumnisse in Bezug auf bestimmte Aspekte des Lebens vorzuhalten. Das können sie beispielshalber dadurch tun, dass sie uns für solche Versäumnisse tadeln. Die Praxis des Zur-Verantwortung-Ziehens kann uns also dabei helfen, Dinge zu ,sehen', die wir zuvor nicht hatten sehen ,können', und auf diese Weise Verantwortung für unsere Denk- und Urteilsfehler zu übernehmen. 
Die Befürworter einer Kontrollbedingung für die Zuschreibung moralischer Verantwortung könnten zwar darauf bestehen, dass verantwortlich für seine Versäumnisse nur sein kann, wer aus eigener Entschlusskraft besser geurteilt oder gehandelt ,haben könnte' oder, etwas weniger anspruchsvoll, wer, auf seinen Fehler hingewiesen, ihn einzusehen und sein Verhalten entsprechend zu ändern vermag. Doch Smith wie Hieronymi zeigen auf, welche Probleme damit verbunden sind, Fähigkeit zum Kriterium für Verantwortungszuschreibung zu machen (vgl. a. Talbert 2012).

Zunächst einmal ist es unerfindlich, weshalb ein normativer Maßstab angesichts einer in Bezug auf ihn bestehenden Unfähigkeit die Flagge streichen sollte. Wenn ich schlecht im Rechnen bin, heißt das nicht, dass die Fehler, die ich mache, akzeptiert oder gebilligt werden sollten. Wenn ich nicht vernünftig für ein Kind sorgen kann, sollte ich keine Elternrolle übernehmen. Warum sollte es sich irgend anders verhalten, wenn ich mich als unfähig erweise, gängige moralische Standards zu erfüllen wenn ich z. B. konstant unzuverlässig oder aggressiv auftrete? Dergleichen sind schwerwiegende Fehler, die schwerwiegende Konsequenzen für die mich umgebenden Menschen zeitigen. Für diese Menschen bleiben es fraglos auch dann - und vielleicht sogar gerade dann - Fehler, wenn ich sie nicht abzustellen weiß, und sie haben allen Grund, den ihnen durch mich bezeigten Mangel an Achtung zu kritisieren, ja mir übelzunehmen - allen Grund auch, ihre Beziehung zu mir zu ändern und bestimmte Formen der Beziehung zu mir gänzlich zu vermeiden. Mit anderen Worten: Sie haben guten Grund, mich als verantwortlich zu behandeln. Wie Hieronymi sagt: „Der moralische Anspruch muss keineswegs im Angesicht der Unfähigkeit des Individuums, ihm gerecht zu werden, weichen. Ein legitimer moralischer Anspruch erfordert nur das Vorliegen der allgemeineren Fähigkeit, in zwischenmenschlichen Beziehungen zu stehen“ (Hieronymi 2007, S. 122).

Hiergegen ließe sich nun einwenden (vgl. Abschn. 4), dass die Fähigkeit, besser zu handeln, aus Gerechtigkeitsgründen doch gegeben sein muss. Wer unfähig ist, moralische Standards einzuhalten, hat keine „faire Chance“, der „Sanktion“ des Zur-Verantwortung-gezogen-Werdens zu entgehen. In seinem bekannten Aufsatz ,Two Faces of Responsibility“ (Watson 2004) hat Gary Watson diesen Einwand durch eine Trennung zwischen Urteilen der „Zuschreibung“ (,attribution“) und solchen der „Zurechnung“ (,accountability“) zu berücksichtigen versucht. Die Zuschreibung von Tugenden und Lastern darf demnach unabhängig von Fragen der Handlungskontrolle erfolgen. Der unverbesserliche Egoist ist somit tatsächlich egoistisch: Sein Denken und Handeln wird von einem unverwechselbaren Bindungs- und Wertungsmuster gesteuert. Derartige moralische Beurteilungen sind insofern angebracht, als der Betreffende über eine gewisse normative Kompetenz verfügt - wenngleich diese Kompetenz sich nicht auf die „Fähigkeit, die richtigen Werte zu erlernen“, erstrecken muss (Watson 2004, S. 282). Was aber die Zurechnung angeht, so ist es eine Forderung der Gerechtigkeit, dass Menschen die Möglichkeit haben, widrige Konsequenzen wie Vergeltung oder Schadensersatzforderungen $\mathrm{zu}$ vermeiden, so dass hierfür das Kriterium der Freiwilligkeit und Handlungskontrolle relevant bleibt. Watson gesteht $\mathrm{zu}$, dass dies noch nicht ausreicht und dass z. B. auch zu den Regeln des gesellschaftlichen Zusammenlebens etwas zu 
sagen wäre: So könnten wir gezwungen sein, um anderer Menschen Rechte oder Ansehens willen dem unverbesserlich Lasterhaften Kosten aufzuerlegen oder Grenzen zu ziehen. Seine Unterscheidung sucht Watson dadurch aufrechtzuerhalten, dass er behauptet, solche Reaktionen verlören dann „ihre übliche Ausdrucksfunktion“ (Watson 2004, S. 281).

Das ist entschieden unbefriedigend. Der unverbesserlich Lasterhafte soll als lasterhaft beurteilt und zugunsten anderer in der Ausübung seiner Laster gezügelt werden. Die Frage wäre, worin eine solche Behandlung sich von derjenigen unterscheiden soll, die wir jemandem zuteilwerden lassen, den wir für sozusagen verbesserlich lasterhaft halten, $d$. h. für fähig, nicht lasterhaft zu sein, obgleich seine Handlungen es sind. (Auch stellt sich die Frage, wie wir eigentlich den einen vom anderen Charakter sollten unterscheiden können ...) Ich sehe hier zwei miteinander zusammenhängende Probleme. Erstens scheint, wie Smith geltend macht, die Art unserer Reaktionen auf Übeltäter bei Watson unterbestimmt zu sein (Smith 2008, S. 375 ff., 2012a). Und zweitens weist dieser Mangel laut Hieronymi (Hieronymi 2004) auf ein Grundproblem des Gerechtigkeitsarguments selber hin. In Anbetracht nämlich der spezifischen Handlungen des Zur-Verantwortung-Ziehens ist es zu simpel, diese als Kosten oder Lasten zuungunsten des Missetäters zu verbuchen (vorsätzlich strafende Reaktionen einmal beiseitegelassen). Denken wir an Tadel, Übelnehmen oder die Tendenz, die Beziehung zum Missetäter auf eine neue Grundlage zu stellen: Tadel beruht auf einem Urteil, wonach jenes Menschen Handlungen und Charakterzüge fehlerhaft sind; insofern in den typischen Gefühlsreaktionen unsere Selbstachtung sich ausdrückt, liegt es nahe, dass wir Verletzungen der Selbstachtung übelnehmen werden; und sich weiterhin auf einen Unzuverlässigen zu verlassen oder eines Anderen Aggression freie Bahn zu lassen, ist offenkundig falsch. All diese Urteile und Reaktionen sind aufgrund der uns widerfahrenen Behandlung angebracht und werden nicht mehr oder weniger angebracht sein, wenn weitere Betrachtungen etwa darüber hinzutreten, ob sie eher dem Wohl oder eher dem Wehe dessen dienen, der uns geschädigt hat, und ob das im größeren Zusammenhang jeweils ,gerecht‘ wäre. Derlei Überlegungen würden die falsche Art von Gründen dafür liefern, Kritik und Übelnehmen zurückzuziehen (oder umgekehrt anzubringen): genauso, wie es falsch wäre, etwas nur deswegen zu glauben, weil mich das glücklich macht.

Dessen ungeachtet behalten natürlich praktische Fragen wie die, wann wir unseren Tadel aussprechen, wie wir unser Übelnehmen beherrschen oder unsere Beziehungen zu anderen gestalten sollten, ihre Bedeutung. Die Antworten werden nicht zuletzt davon abhängen, zu welcher Reaktion auf unsere eigene Reaktion die andere Seite fähig oder geneigt scheint. Andererseits dürfen wir nicht vergessen, wie komplex unsere Praxis des Zur-Verantwortung-Ziehens ist und wie sie mit den vielen anderen Arten von Gründen zusammenspielen muss, die unser Miteinander regeln (vgl. bes. Smith 2007). Das heißt, die vermeintlich so einfache Frage: „Ist jemand für seine Laster oder Missetaten verantwortlich?" kann uns hier keinen ausreichenden Leitfaden bieten. Wenn wir alle anderweitigen Gründe dafür, dass wir einander einen bestimmten Umgang schulden, beiseiteschieben, überdehnen wir 
das Verantwortungsprinzip, so dass es seiner Bindekraft verlustig geht und endlich kaum verwunderlich - als ungerecht oder sonstwie fragwürdig erscheint.

Zusammenfassend lässt sich sagen, dass eines Menschen Kontrolle über seine Handlungen in zwei zentralen Hinsichten begrenzt ist. (i) Urteile und Einstellungen sind zumindest im Normalfall unserer Kontrolle schlechterdings entzogen: Wir können nicht ,nach Belieben' glauben, beabsichtigen, übelnehmen, fürchten oder lieben. Solche Einstellungen sind eher als Manifestationen unserer rationalen und evaluativen Tätigkeit zu verstehen. Sofern wir eine Kontrolle ausüben, liegt sie „behind the lens, so to speak“ (Hieronymi 2008, S. 371). Das heißt, unsere Gedanken kontrollieren wir, indem wir eben denken; entsprechend kontrollieren wir auch unsere wertende Tätigkeit, indem wir ihr obliegen, indem wir also urteilen, abwägen und allmählich lernen, was für uns erfüllend ist und was von anderen geschätzt wird. Betrachten wir das Vermögen, verantwortlich zu handeln, insgesamt, so scheint die bewusste Ausübung von Kontrolle, etwa bei vorsätzlichen Handlungen oder bei dem Versuch, eine bestimmte Einstellung zu ändern, mehr die Spitze des Eisbergs denn der basale oder paradigmatische Fall zu sein. (ii) Wir sind, wie Fischer und Ravizza unterstreichen, für Gründe nur ,,mäßig“ empfänglich. Es wäre deshalb unstimmig, die Verantwortungspraxis damit zu rechtfertigen, dass wir fähig seien, richtigzuliegen. Unser Denken und Urteilen ist, welche Eigenschaften der Fähigkeit dazu sonst auch immer zukommen mögen, fehlbar. Es wäre ein Irrtum, anzunehmen, unsere Fehler ließen sich unter Kontrolle bringen. Ohne Zweifel gibt es absichtliche Versäumnisse und vorsätzliches Unrecht, doch handelt es sich dabei um Ausnahmen, nicht die Regel. Ihnen zugrunde liegen Aufmerksamkeitsmuster, eine evaluative Grundausstattung, die Neigung, manche Menschen und Dinge wichtiger als andere zu nehmen - lauter Voraussetzungen, die einen Menschen schlimmstenfalls davon abhalten, Gründe für andere Denk- und Handlungsweisen anzuerkennen, und die ihn noch im besten Falle gegen spezifische Gründe oder Werte blind machen können. Die jeweiligen Grenzen des Handlungsvermögens eines Menschen geben Anderen mehr und nicht weniger Grund zur Überprüfung seines Handelns und Wertens, zumindest so lange, wie sie nicht sämtliches Vertrauen in seine Fähigkeit, Normen für unser Zusammenleben in der Welt zu bedenken und beurteilen, verloren haben.

Aus all diesen Gründen legen sowohl Smith als Hieronymi den Akzent nicht auf die Wahl, sondern auf das Urteil. Unsere Fähigkeit zum vorsätzlichen Handeln und zur Selbstkontrolle ist zwangsläufig begrenzt. Doch niemand kann es vermeiden, im Laufe seines Lebens eine Haltung zu der Frage einzunehmen, was für ihn wichtig und auf welche Grundlage sein Zusammenleben mit anderen zu stellen sei. Ob er seine Wertungen explizit be- und überdenkt oder nicht, sie finden sich auf jeden Fall implizit in seinem Denken und Tun und haben tief greifende Folgen für andere, die mit ihm interagieren. Ein eingehendes Verständnis dessen verlangt erstens nach einer Konzeption von (verantwortlicher) Tätigkeit, die nicht auf der Idee eines separaten Kontrolleurs aufbaut, und zweitens - auch wenn dies bei den hier erörterten Autoren nur eben angedeutet wird (Hieronymi 2014) - nach einer Konzeption von wechselseitiger Anerkennung, aufgrund deren verantwortungsfähige Personen einander - und sich selbst - als Urheber jener Tätigkeit sowie als Träger entsprechender Verpflichtungen verstehen. 


\section{$8 \quad$ Zusammenfassung}

Alle hier betrachteten Autoren liefern wichtige Beiträge zum Verständnis des Zusammenhangs von Verantwortung, Rationalität und Urteil. Für die Verknüpfung der Verantwortung mit unserem Denk- und Urteilsvermögen führen sie zwingende Argumente an. Auch bestärken sie uns in der Annahme, dass dieses Vermögen mit der kausalen Ordnung der Welt kompatibel sei, wenngleich es distinkter normativer Kategorien bedürfe, um sichtbar für uns zu werden.

Die inkompatibilistischen Bedenken speisen sich nach meinem Eindruck nach wie vor aus zwei großen Problemkreisen. Zum einen ist die reale Praxis des ZurVerantwortung-Ziehens - mit der allerdings bedeutenden Ausnahme des Bestrafens lange Zeit fast völlig ausgeblendet worden und erst in den letzten paar Jahren zu einem Gegenstand der Forschung avanciert (nicht zuletzt bei Smith 2007, 2012b und Hieronymi 2004). Manche Autoren sagen offen, dass sie Verantwortung schon deshalb nicht für kompatibilistisch begründbar halten, weil sie sich an einem Verantwortungsbegriff orientieren, den menschliche Wesen prinzipiell nicht ausfüllen können; so etwa Galen Strawson mit seiner „Himmel-und-Höllen-Verantwortung“ (,heaven-and-hell responsibility“) (z. B. Strawson 2008). Allgemein und mit Peter Strawson (Strawson 1962) gesprochen: Wenn wir uns auf die abstrakte Frage konzentrieren, ob Menschen verantwortlich sind oder nicht bzw. ob sie gerechterweise zur Verantwortung gezogen werden dürfen (wo dies simplistisch als Übel oder Beschwernis veranschlagt wird), verlieren wir die mit der Verantwortungspraxis verbundenen Beziehungen und Interaktionen aus dem Blick. Verlieren wir aber die mit unserem Gegenstand verbundenen Aspekte aus dem Blick, wird der Gegenstand selbst begreiflicherweise umso rätselhafter.

Zum anderen sind, wie mehrfach erwähnt, auch die grundlegenderen Begriffe der rationalen Handlungsfähigkeit und des moralischen Vermögens noch längst nicht ausreichend untersucht. Gemessen an der großen Zahl von Debatten zwischen Kompatibilisten und Befürwortern eines metaphysischen freien Willens ist erstaunlich wenig über den Vermögens- bzw. Fähigkeitsbegriff und insbesondere über unsere Denk- und Urteilsfähigkeit zutag gefördert worden. J. L. Austin hat einmal bemerkt: „Immer wenn wir gerade geglaubt hatten, wir hätten ein Problem gelöst, lugt besonders gern das Können wieder hervor und grinst zu uns herauf gleichwie der [Kierkegaardsche] Frosch, der als Bodensatz am Grund des Bierkrugs hockt" (Austin 1961, S. 231). Man kann sich des Eindrucks nicht erwehren, dass der Frosch immer noch da ist und zu uns emporgrinst oder uns Grimassen schneidet.

Diese beiden Problemkreise überschneiden einander auf interessante Weise. Wie verschiedentlich hier angemerkt, haben wir Grund zu der Annahme, dass die Praxis des Zur-Verantwortung-Ziehens in einem konstitutiven Zusammenhang mit unserer Fähigkeit steht, als rationale und verantwortliche Personen $\mathrm{zu}$ handeln, solche Personen also zu sein. Ohne die Gesellschaft anständiger Menschen, die bereit und imstande sind, uns unter anständigen Bedingungen zur Verantwortung zu ziehen, erweisen sich unsere Fähigkeiten zum rationalen Denken und moralischen Urteilen häufig als allzu schwach. Das soll nicht heißen, dass Menschen für ihre Mitwirkung an verbreiteten Übeln nicht $\mathrm{zu}$ tadeln oder sonstwie verantwortlich $\mathrm{zu}$ machen 
wären. Es ist aber ein Hinweis darauf, dass (scheinbar) rein individuelle Fähigkeiten auch eine soziale Dimension haben und dass sich dies an der Praxis des ZurVerantwortung-Ziehens ablesen lässt (Vargas 2013).

Zusätzlich ließe sich noch darauf hinweisen, dass verantwortungsfähige Menschen dieses gesellschaftliche Moment auch auf ihrer eigenen inneren Bühne inszenieren, indem sie die Perspektiven und möglichen Reaktionen anderer auf ihre Denk- und Handlungsweisen durchspielen. Fischer und Ravizza stellen zu Recht die Übernahme von Verantwortung für das eigene Handeln als individuelle Fähigkeit und Pflicht ineins heraus. Wir könnten auch sagen: Für die Fähigkeit zum verantwortlichen Handeln ist es grundlegend, dass jeder Einzelne sich selber zur Verantwortung zu ziehen lerne; oder noch stärker: Dies zu tun, ist Teil von Rationalität selbst (vgl. Korsgaard 2009). Ohne die Fähigkeit, die Perspektive anderer einzunehmen und die Möglichkeit zuzulassen, dass sie uns in unserem Handeln ein wertvolles Korrektiv bietet, wäre kein Mensch je in der Lage, an der sozialen Praxis des ZurVerantwortung-Ziehens teilzunehmen. Diese wäre dann lediglich eine Form der Zwangsausübung - bestenfalls die gut gemeinte, doch manipulative „Droh-Ökonomie“ (,economy of threats“) der Utilitaristen, schlimmstenfalls die Forderung nach Anpassung in einem unterdrückerischen oder ungerechten Sozialgefüge.

$\mathrm{Zu}$ welchem Verständnis all dieser Aspekte wir dereinst auch gelangen werden, der thematische Rahmen sollte mit dem vorliegenden Beitrag abgesteckt sein. Die Praxis des Zur-Verantwortung-Ziehens hängt mit der spezifisch menschlichen (und daher unvermeidlich fragilen und fehlbaren) Fähigkeit zum moralischen Denken und Handeln zusammen. Die Frage der Rationalität dieser Praxis selbst konnte hier nur gestreift werden. Doch sollte immerhin deutlich geworden sein, dass die Praxis des Zur-Verantwortung-Ziehens in einer engen Beziehung zu jener des VerantwortungÜbernehmens steht und dass beide eine wichtige Rolle bei der Aufrechterhaltung rationaler Handlungsfähigkeit sowie moralischer Gemeinschaft spielen.

\section{Literatur}

Adams, Robert Merrihew. 1985. Involuntary sins. The Philosophical Review 94:3-31.

Austin, J. L. 1961. Ifs and cans. In his Philosophical Papers, 153-180. Oxford: Clarendon.

Benson, Paul. 1996. Review of R Jay Wallace, Responsibility and the moral sentiments. Journal of Philosophy 93:574-578.

Boyle, Matthew. 2013. Active belief. Canadian Journal of Philosophy 39(Suppl. 1):119-147.

Boyle, Matthew. 2011. ,Making up your mind“ and the activity of reason. Philosophers' Imprint 11(17).

Coates, D. Justin, und Neal A. Tognazzini, Hrsg. 2012. Blame: Its nature and norms. New York: Oxford University Press.

Cullity, Garrett. 1997. Review of R Jay Wallace, Responsibility and the moral sentiments. Mind 106:803-807.

Fischer, John Martin. 1982. Responsibility and control. Journal of Philosophy 89:24-40.

Fischer, John Martin. 1987. Responsiveness and Moral Responsibility. In Responsibility, character and the emotions, Hrsg. Ferdinand Schoeman, 81-106. Cambridge: Cambridge University Press (nachgedr. als Kap. 3 in Fischer 2006:63-83).

Fischer, John Martin. 1994. The metaphysics of free will: An essay on control. Oxford: Blackwell. 
Fischer, John Martin. 2006. My way: Essays on moral responsibility. New York: Oxford University Press.

Fischer, John Martin. 2012. Semicompatibilism and its rivals. Journal of Ethics 16:117-43.

Fischer, John Martin, und Mark Ravizza. 1998. Responsibility and control: A theory of moral responsibility. Cambridge: Cambridge University Press.

Fischer, John Martin, Robert Kane, Derk Pereboom, und Manuel R. Vargas. 2007. Four views on free will. Malden: Wiley-Blackwell.

Frankfurt, Harry G. 1969. Alternate possibilities and moral responsibility. The Journal of Philosophy 66:829-839.

Frankfurt, Harry G. 1971. Freedom of the will and the concept of a person. The Journal of Philosophy 68:5-20.

Hieronymi, Pamela. 2004. The force and fairness of blame. Philosophical Perspectives 18:115-148.

Hieronymi, Pamela. 2006. Controlling attitudes. Pacific Philosophical Quarterly 87:45-74.

Hieronymi, Pamela. 2007. Rational capacity as a condition on blame. Philosophical Books 48: $109-123$.

Hieronymi, Pamela. 2008. Responsibility for believing. Synthese 161:357-373.

Hieronymi, Pamela. 2014. Reflection and responsibility. Philosophy and Public Affairs 42:3-41.

Kane, Robert. 2002. Responsibility, reactive attitudes and free will: Reflections on Wallace's theory. Philosophy and Phenomenological Research 64:693-698.

Korsgaard, Christine M. 2009. Self-constitution: Agency, identity, and integrity. Oxford: Oxford University Press.

Korsgaard, Christine M. 2010. The activity of reason. Proceedings and Addresses of the American Philosophical Association 83:23-43.

Lenman, James. 2006. Compatibilism and contractualism: The possibility of moral responsibility. Ethics 117:7-31.

Levy, Neil. 2005. The good, the bad and the blameworthy. Journal of Ethics \& Social Philosophy 1: $2-16$.

Moran, Richard. 2012. Self-knowledge, ,transparency', and the forms of activity. In Introspection and consciousness, Hrsg. Declan Smithies und Daniel Stoljar, 211-236. Oxford: Oxford University Press.

Otsuka, M. 1998. Incompatibilism and the avoidability of blame. Ethics 108:685-701.

Ripstein, Arthur. 2004. Justice and responsibility. Canadian Journal of Law and Jurisprudence 17:361-386.

Scanlon, T.M. 1998. What we owe to each other. Cambridge, MA: Belknap Press.

Sher, George. 2005. In praise of blame. Oxford: Oxford University Press.

Sher, George. 2005. Out of control. Ethics 116:285-301.

Smart, J.J.C. 1961. Free-will, praise and blame. Mind 70:291-306.

Smith, Angela M. 2004. Conflicting attitudes, moral agency, and conceptions of the self. Philosophical Topics 32:331-352.

Smith, Michael. 2004. Rational capacities, or: How to distinguish recklessness, weakness, and compulsion. In his Ethics and the a priori, 114-35. Cambridge: Cambridge University Press.

Smith, Angela M. 2005. Responsibility for attitudes: Activity and passivity in mental life. Ethics 115:236-271.

Smith, Angela M. 2007. On being responsible and holding responsible. The Journal of Ethics 11:465-484.

Smith, Angela M. 2008. Control, responsibility, and moral assessment. Philosophical Studies $138: 367-392$.

Smith, Angela M. 2012a. Attributability, answerability, and accountability: In defense of a unified account. Ethics 122:575-589.

Smith, Angela M. 2012b. Moral blame and moral protest. In Coates \& Tognazzini, Hrsg. 2012.

Strawson, P. F. 1962. Freedom and Resentment. Proceedings of the British Academy 48:1-25; dt.: Freiheit und Übelnehmen. In Seminar: Freies Handeln und Determinismus, Hrsg. Ulrich Pothast, 201-233. Frankfurt a. M.: Suhrkamp, 1978. 
Strawson, Galen. 2008. The impossibility of ultimate moral responsibility. In his Real materialism and other essays, 319-331. Oxford: Clarendon.

Talbert, Matthew. 2012. Moral competence, moral blame, and protest. The Journal of Ethics $16: 89-109$.

Vargas, Manuel. 2013. Building better beings: A theory of moral responsibility. Oxford: Oxford University Press.

Wallace, R. Jay. 1994. Responsibility and the moral sentiments. Cambridge, MA: Harvard University Press.

Wallace, R. Jay. 2002. Replies. Philosophy and Phenomenological Research 94:707-727.

Watson, Gary. 1975. Free Agency. The Journal of Philosophy 72:205-220.

Watson, Gary. 2004. Two faces of responsibility. In his Agency and answerability, 260-288. New York: Oxford University Press.

Williams, Bernard. 1993. Moral incapacity. Proceedings of the Aristotelian Society 93:59-70.

Wolf, Susan. 1980. Asymmetrical freedom. The Journal of Philosophy 77:151-166.

Wolf, Susan. 1987. Sanity and the metaphysics of responsibility. In Responsibility, character and the emotions, Hrsg. Ferdinand Schoeman, 46-62. Cambridge: Cambridge University Press.

Wolf, Susan. 1990. Freedom within reason. New York: Oxford University Press.

Wolf, Susan. 2005. Freedom within reason. In Personal autonomy: New essays on personal autonomy and its role in contemporary moral philosophy, Hrsg. Taylor James Stacey, 258-274. Cambridge: Cambridge University Press. 